\title{
Ergodicity and Gaussianity for spherical random fields
}

\author{
Domenico Marinucci ${ }^{1, a)}$ and Giovanni Peccati ${ }^{2}$ \\ ${ }^{1}$ Dipartimento di Matematica, Università di Roma Tor Vergata, Roma 00133, Italy \\ ${ }^{2}$ Unité de Recherche en Mathématiques, Université du Luxembourg, L-1359 Luxembourg
}

(Received 12 November 2009; accepted 2 February 2010; published online 7 April 2010)

\begin{abstract}
We investigate the relationship between ergodicity and asymptotic Gaussianity of isotropic spherical random fields in the high-resolution (or high-frequency) limit. In particular, our results suggest that under a wide variety of circumstances the two conditions are equivalent, i.e., the sample angular power spectrum may converge to the population value if and only if the underlying field is asymptotically Gaussian in the high-frequency sense. These findings may shed some light on the role of cosmic variance in cosmic microwave background radiation data analysis.

(C) 2010 American Institute of Physics. [doi:10.1063/1.3329423]
\end{abstract}

\section{INTRODUCTION AND BACKGROUND}

\section{A. Overview}

The usual framework for proving asymptotic results in probability (for instance, central limit theorems or laws of large numbers) lies within the so-called large sample paradigm, according to which more and more (independent or weakly dependent) random variables are generated, and the limiting behavior of some functionals of these variables (e.g., averages or empirical moments) is studied.

Physical applications, however, are prompting the development of a stochastic asymptotic theory of a rather different nature, where the indefinite repetition of a single experience is no longer available, and one relies instead on observations of the same (fixed) phenomenon with higher and higher degrees of resolution.

One crucial instance of this situation appears when dealing with the statistical analysis of random fields indexed by compact manifolds, the quintessential example being provided by the case of the sphere $S^{2}$. Indeed, we are especially concerned with issues arising from the analysis of the cosmic microwave background (CMB) radiation, a theme that is currently at the core of physical and cosmological research (see, for instance, Refs. 12 and 19 for textbook references and Refs. 38, 37, and 20 for further discussions around the latest experimental data).

It is well known that the $\mathrm{CMB}$ is a relic electromagnetic radiation providing a snapshot of the Universe at the so-called age of recombination, i.e., at the era when electrons in the primordial fluid arising from the Big Bang were captured by protons to form stable hydrogen atoms. Since the cross section of hydrogen atoms is much smaller than that for free electrons, after recombination photons can be viewed as diffusing freely across the Universe (to first order approximations). According to the latest experimental evidence, this has occurred some $3.7 \times 10^{5} \mathrm{yr}$ after the Big Bang, i.e., $13.7 \times 10^{9} \mathrm{yr}$ from the current epoch. Several experiments have been devoted to collecting extremely refined observations of the $\mathrm{CMB}$, the leading role being played by the currently ongoing NASA mission Wilkinson microwave anisotropy probe (WMAP) (launched in 2001, see http://map.gsfc.nasa.gov/) and the ESA mission Planck, which is just now starting to operate after the launch on May 14, 2009 (see http://www.sciops.esa.int/).

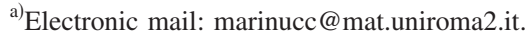


From a mathematical point of view, the CMB can be regarded as a single realization of an isotropic, zero-mean, finite variance spherical random field, for which the following spectral representation holds (see, e.g., Ref. 1 or Ref. 21):

$$
T(x)=\sum_{l=0}^{\infty} \sum_{m=-l}^{l} a_{l m} Y_{l m}(x), \quad x \in S^{2} .
$$

Here, the collection

$$
\left\{Y_{l m}: l \geq 0, m=-l, \ldots, l\right\}
$$

stands for the usual triangular array of spherical harmonics, which are well known to provide a complete orthonormal system for the $L^{2}\left(S^{2}\right)$ space of square-integrable functions (with respect to Lebesgue measure) on the sphere (see Refs. 39, 42, and 43). In a loose sense, we can say that the frequency parameter $l$ is related to a characteristic angular scale, say $\vartheta_{l}$, according to the relationship $\vartheta_{l} \simeq \pi / l$. The (random) triangular array of spherical harmonic coefficients $\left\{a_{l m}: l \geq 0, m=\right.$ $-l, \ldots, l\}$ is such that $E a_{l m}=0$ and $E a_{l m} \bar{a}_{l^{\prime} m^{\prime}}=C_{l} \delta_{l}^{l^{\prime}} \delta_{m}^{n^{\prime}}$, the bar denoting complex conjugation and $\delta_{a}^{b}$ indicating the Kronecker delta function. The non-negative sequence $\left\{C_{l}: l \geq 0\right\}$ (not depending on $m$-see Ref. 27 as well as Sec. I B) is the angular power spectrum of the spherical field (see, for instance, Refs. 2 and 3).

As recalled above, our work deals with asymptotic issues, where the expression "asymptotic" has to be understood in the high-resolution (or high-frequency) sense. This means that we focus on the behavior of the Fourier components

$$
T_{l}(x):=\sum_{m=-l}^{l} a_{l m} Y_{l m}(x), \quad x \in S^{2}, \quad l \geq 0,
$$

associated with a fixed spherical field, as the frequency $l$ grows larger and larger [plainly, each $T_{l}$ is the projection of the field $T$ into the orthogonal subspace of $L^{2}\left(S^{2}\right)$ spanned by the spherical harmonics $\left.\left\{Y_{l m}: m=-l, \ldots, l\right\}\right]$. Note that this is the typical framework faced by experimentalists handling satellite missions as those mentioned above. Indeed, these missions are observing the same (unique) realization of our Universe on the so-called last scattering surface; more recent and more sophisticated experiments are then characterized by higher and higher frequencies (smaller and smaller scales) being observed. For instance, for the pioneering CMB mission COBE in 1989-1992 (which led to the Nobel Prize for Smoot and Mather in 2006), only frequencies of the order of a few dozens were recorded (i.e., scales of several degrees), a limit which was raised to few hundreds by WMAP (i.e., approximately a quarter of degree) and is expected to grow to a few thousands with Planck (i.e., a few arc min). Note also that $T_{l}$ is clearly a random eigenfunction of the spherical Laplacian $\Delta_{S^{2}}$; as such, its geometric properties (especially the behavior of its nodal sets) have been widely investigated over the past few years (see Refs. 44 and 45 and references therein).

The principal goal of this paper is to enlighten some partial new connections between two high-resolution characterizations of spherical fields, that is, ergodicity and asymptotic Gaussianity. Roughly speaking (formal details are given in Secs. I B and I C), one says that the spherical field $T$ is ergodic if the empirical version of the power spectrum of $T$ [see formula (3) below] can be used as a consistent estimator of the sequence $\left\{C_{l}\right\}$ (at least for high values of $l$ ). On the other hand, we say that $T$ is asymptotically Gaussian, whenever suitably normalized versions of the frequency components of $T_{l}$ exhibit Gaussian fluctuations for high values of $l$. As discussed below, these two notions are tightly connected whenever one deals with fields having an isotropic (or, equivalently, rotationally invariant) law. See also Refs. 7, 10, 15, 17, 18, 23, 24, 26, 28, 29, 33, 34, 36, 40, and 41 for related results.

Remark: For the rest of the paper, every random object is defined on a suitable (common) probability space $(\Omega, \mathcal{F}, P)$. 


\section{B. High-frequency ergodicity}

In what follows, we shall consider a real-valued random field $T=\left\{T(x): x \in S^{2}\right\}$ indexed by the sphere $S^{2}$. The random field $T$ satisfies the following basic assumptions: (i) the law of $T$ is isotropic, that is, $T$ has the same law as $x \mapsto T(g x)$ for every rotation $g \in \mathrm{SO}$ (3) [here, we select the canonical action of $\mathrm{SO}(3)$ on $S^{2}$; (ii) $T$ is square integrable and centered. Under assumptions (i) and (ii), the harmonic expansion (1) takes place, both in $L^{2}(P)$ (for fixed $x$ ) and in the product space $L^{2}\left(\Omega \times S^{2}, P \otimes d \lambda\right)$, where $\lambda$ stands for the Lebesgue measure. Note that the last claim hinges on the fact that one can regard $T$ as an application of the type $T: \Omega \times S^{2}$ $\rightarrow \mathbb{R}:(\omega, x) \mapsto T(\omega, x)$. As anticipated in Sec. I A, another useful property of $T$ (easily deduced from isotropy-see, e.g., Ref. 27) is that the harmonic coefficients $a_{l m}$ are such that the power spectrum associated with $T$, defined as the collection $\left\{C_{l}: l=0,1, \ldots\right\}$ (with $C_{l}=E\left|a_{l m}\right|^{2}$ ), depends uniquely on the frequency index $l$.

In physical experiments (for instance, when measuring the CMB radiation), the power spectrum of a given spherical field is usually unknown. For this reason, a key role is played by its empirical counterpart (called the empirical power spectrum—see, for instance, Refs. 13 and 35), which is given by

$$
\hat{C}_{l}=\frac{1}{2 l+1} \sum_{m=-l}^{l}\left|a_{l m}\right|^{2}, \quad l=0,1,2, \ldots .
$$

An important issue to be addressed is therefore to establish conditions under which the distance between the quantities $\hat{C}_{l}$ and $C_{l}$ converge to zero (in a sense that is defined below) when $l \rightarrow \infty$, that is, when higher and higher frequencies of the expansion (1) are available to the observer. Although the asymptotic behavior of spectrum estimators has been very deeply investigated for stochastic processes in Euclidean domains and under large sample asymptotics (see, for instance, Refs. 6, 21, and 47), only basic results are known in the high-resolution setting.

For instance, it is immediate that the finite variance of $T$ entails that for every $x \in S^{2}$,

$$
E T(x)^{2}=\sum_{l \geq 0} \frac{(2 l+1)}{4 \pi} C_{l}<\infty,
$$

from which one deduces that $C_{l} \rightarrow 0$ and also

$$
\sum_{l \geq 0} E \hat{C}_{l}=\sum_{l \geq 0} C_{l}<\infty
$$

By reasoning as in the proof of the Borel-Cantelli lemma, we therefore infer that for any $\varepsilon>0$,

$$
P\left\{\lim _{l \rightarrow \infty} \sup \hat{C}_{l}>\varepsilon\right\} \leq \lim _{l \rightarrow \infty} \sum_{\ell \geq l} P\left\{\hat{C}_{\ell} \geq \varepsilon\right\} \leq \lim _{l \rightarrow \infty} \frac{1}{\varepsilon} \sum_{\ell \geq l} C_{\ell}=0
$$

yielding, in turn, that both $\hat{C}_{l}$ and $\left|\hat{C}_{l}-C_{l}\right|$ almost surely converge to zero as $l \rightarrow \infty$. Plainly, since this result does not provide any information about the magnitude of the ratio $\left|\hat{C}_{l}-C_{l}\right| / C_{l}$, it is virtually useless for statistical applications. In particular, one cannot conclude from (4) that the estimation of $C_{l}$ based on $\hat{C}_{l}$ is consistent in a satisfactory statistical sense.

Starting from these considerations, one sees that it is indeed necessary to focus on the normalized quantities, such as the sequence

$$
\tilde{C}_{l}=\frac{1}{2 l+1} \sum_{m=-l}^{l} \frac{\left|a_{l m}\right|^{2}}{C_{l}}=\frac{\hat{C}_{l}}{C_{l}}, \quad l \geq 0 .
$$

Note that $E \widetilde{C}_{l}=1$ and also that the coefficient $\widetilde{C}_{l}$ is not observable (whereas $\hat{C}_{l}$ is). The sequence $\left\{\widetilde{C}_{l}: l \geq 0\right\}$ can be used in order to meaningfully evaluate the asymptotic performance of any 
statistical procedure based on $\hat{C}_{l}$. The following definition uses the coefficients $\widetilde{C}_{l}$ in order to define ergodicity.

Definition 1: (High-frequency ergodic) Let T be an isotropic, finite variance spherical random field with angular power spectrum $\left\{C_{l}: l \geq 0\right\}$. We shall say that $T$ is high-frequency ergodic (HFE) - or ergodic in the high-frequency sense) if and only if

$$
\lim _{l \rightarrow \infty} E\left\{\tilde{C}_{l}-1\right\}^{2}=\lim _{l \rightarrow \infty} E\left\{\frac{\hat{C}_{l}}{C_{l}}-1\right\}^{2}=0 .
$$
1.

Condition (6) implies, of course, that $\widetilde{C}_{l}=\hat{C}_{l} / C_{l}$ converges in probability toward the constant

Remark: The term "high-frequency consistency" could provide an alternative definition for property (6) However, in the statistical literature, consistency is usually viewed as a property of a sequence of estimators, whereas here we deal with a property of the field $T$ so that we find the term ergodicity more suitable. Note that by Parseval identity

$$
\int_{S^{2}} T_{l}^{2}(x) d x=\sum_{l m}\left|a_{l m}\right|^{2}=(2 l+1) \hat{C}_{l}
$$

and therefore

$$
\widetilde{C}_{l}=\frac{\int_{S^{2}} T_{l}^{2}(x) d x}{E T_{l}^{2}} .
$$

Hence, the HFE property is stating that the realized mean of the random function $T_{l}^{2}(x)$ (averaged over $S^{2}$ ) is converging to the population mean $E T_{l}^{2}$ (averaged over the probability measure). In this sense the term HFE (in the mean-square sense) seems fully appropriate.

\section{Ergodicity of Gaussian fields (and associated Gaussian fluctuations)}

As an illustration (and for future reference) we now test Definition 1 under the additional assumption that $T$ is Gaussian. In this case, it is readily seen that for every $l \geq 1$, the components of the vector $\left\{a_{l m}: m=1, \ldots, l\right\}$ are complex valued and independent. Moreover, the random quantities $a_{l 0} / \sqrt{C_{l}}, \sqrt{2} \operatorname{Re}\left(a_{l m}\right) / \sqrt{C_{l}}$, and $\sqrt{2} \operatorname{Im}\left(a_{l m}\right) / \sqrt{C_{l}}(m=0, \ldots, l)$ are independent and identically distributed $N(0,1)$ random variables [these facts are well known (see, e.g., Refs. 2 and 27 and references therein)]. It is now easy to prove that

$$
\tilde{C}_{l}=\frac{1}{2 l+1} \sum_{m=-l}^{l} \frac{\left|a_{l m}\right|^{2}}{C_{l}} \rightarrow 1
$$

in every norm $L^{p}, p \geq 1$. Indeed, since $a_{l 0}^{2} / C_{l} \sim \chi_{1}^{2}$ and the set $\left\{2 a_{l m}^{2} / C_{l}: m=1, \ldots, l\right\}$ is composed of independent and identically distributed (i.i.d.) $\chi_{2}^{2}$ random variables independent of $a_{l 0}$ (here, $\chi_{n}^{2}$ denotes a standard chi-square distribution with $n$ degrees of freedom),

$$
E\left\{\widetilde{C}_{l}-1\right\}^{2}=\frac{1}{(2 l+1)^{2}} E\left[\frac{a_{l 0}^{2}}{C_{l}}-1+2\left\{\sum_{m=1}^{l} \frac{\left|a_{l m}\right|^{2}}{C_{l}}-1\right\}\right]^{2}=\frac{2}{2 l+1} \rightarrow 0,
$$

and one can use the fact that for polynomial functionals of a Gaussian field of fixed degree, all $L^{p}$ topologies coincide.

We shall now provide (see the forthcoming Proposition 2) a CLT that is naturally associated with the convergence described in (7). Note that, instead of using the classic Berry-Esseen results (see, e.g., Ref. 14), we rather apply some recent estimates (proved in Refs. 31 and 32 by means of infinite-dimensional Gaussian analysis and the so-called "Stein's method" for probabilistic ap- 
proximations) allowing one to compare, for fixed $l$, the total variation distance between the law of the normalized random variable

$$
\sqrt{\frac{2 l+1}{2}}\left\{\frac{\hat{C}_{l}}{C_{l}}-1\right\}=\sqrt{\frac{2 l+1}{2}}\left\{\tilde{C}_{l}-1\right\},
$$

and that of a standard Gaussian random variable. Recall that the total variation distance between the laws of two real-valued random variables $X$ and $Y$ is given by

$$
d_{\mathrm{TV}}(X, Y)=\sup _{A}|P(X \in A)-P(Y \in A)|,
$$

where the supremum runs over all Borel sets $A$.

Proposition 2: Let $N(0,1)$ denote a centered standard Gaussian random variable. Then, for all $l \geq 0$ we have

$$
d_{\mathrm{TV}}\left(\sqrt{\frac{2 l+1}{2}}\left\{\frac{\hat{C}_{l}}{C_{l}}-1\right\}, N(0,1)\right) \leq \sqrt{\frac{8}{2 l+1}},
$$

so that, in particular, as $l \rightarrow \infty$,

$$
\sqrt{\frac{2 l+1}{2}}\left\{\widetilde{C}_{l}-1\right\} \rightarrow N(0,1) .
$$

Proof: We have

$$
\begin{aligned}
\sqrt{\frac{2 l+1}{2}}\left\{\frac{\hat{C}_{l}}{C_{l}}-1\right\} & =\frac{1}{\sqrt{2(2 l+1)}}\left\{\frac{a_{l 0}^{2}}{C_{l}}+\sum_{m=1}^{l} 2 \frac{\left\{\operatorname{Re} a_{l m}\right\}^{2}+\left\{\operatorname{Im} a_{l m}\right\}^{2}}{C_{l}}-(2 l+1)\right\} \\
& =\frac{1}{\sqrt{(2 l+1)}}\left\{\sum_{m=1}^{2 l+1} \frac{\left(x_{l m}^{2}-1\right)}{\sqrt{2}}\right\},
\end{aligned}
$$

where $\left\{x_{l m}\right\}$ are a triangular array of i.i.d. standard Gaussian random variables. Standard calculations yield that

$$
\operatorname{cum}_{4}\left\{\frac{\sqrt{2}}{\sqrt{(2 l+1)}}\left[\sum_{m=1}^{2 l+1} \frac{\left(x_{l m}^{2}-1\right)}{2}\right]\right\}=\frac{12}{2 l+1},
$$

where $\mathrm{cum}_{j}$ stands for the $j$ th cumulant. Now recall that in Ref. 32 it is proved that for every zero mean and unit variance random variable $F_{q}$ that belongs to the $q$ th Wiener chaos associated with some Gaussian field $(q \geq 2)$, the following inequality holds:

$$
d_{\mathrm{TV}}\left(F_{q}, N(0,1)\right) \leq 2 \sqrt{\frac{q-1}{3 q}} \sqrt{\operatorname{cum}_{4}\left(F_{q}\right)} .
$$

The result now follows immediately since each variable $\sqrt{[(2 l+1) / 2]}\left\{\hat{C}_{l} / C_{l}-1\right\}$ has unit variance and is precisely an element of the second Wiener chaos associated with $T$.

It is simple to verify numerically that the convergence (9) takes place rather fast. For instance, for $l=100$, the bound in the total variation is of the order of $2 \%$, while for $l=1000$, we deduce an order of $0.6 \%$.

We stress that the previous results heavily rely on the Gaussian assumption and cannot be easily extended to the framework of non-Gaussian and isotropic spherical fields. The main reason supporting this claim is contained in Refs. 2 and 3 where it is shown that, under isotropy, the coefficients $a_{l m}$ are independent if and only if the underlying field is Gaussian and despite the fact that they are always uncorrelated by construction. In other words, sampling independent, non- 
Gaussian random coefficients to generate maps according to (1) will always yield an anisotropic random field. The dependence structure among the coefficients $\left\{a_{l m}\right\}$ is, in general, quite complicated, albeit it can be neatly characterized in terms of the group representation properties of $\mathrm{SO}(3)$ (see Refs. 25 and 27). In view of this, to derive any asymptotic result for $\hat{C}_{l}$ under non-Gaussianity assumptions for $T$ is by no means trivial and still almost completely open for research.

\section{High-frequency Gaussianity}

A different form of asymptotic theory has been addressed in an apparently unrelated stream of research, for instance, in Ref. 25.

Definition 3: (High-frequency Gaussian) Let $T(x)$ be an isotropic, finite variance spherical random field, and recall notations (1) and (2). We say that $T(x)$ is high-frequency Gaussian (HFG) whenever

$$
\frac{T_{l}(x)}{\sqrt{\operatorname{Var}\left\{T_{l}(x)\right\}}} \stackrel{\text { law }}{\rightarrow} N(0,1), \quad \text { as } l \rightarrow \infty
$$

for every fixed $x \in S^{2}$.

Remark: It is more delicate to define HFG involving convergence in the sense of finite dimensional distributions. Indeed, in Ref. 25 it is shown that even if relation (10) holds, the finite-dimensional distributions of the order of $\geq 2$ of the field $x \mapsto T_{l}(x) / \sqrt{\operatorname{Var}\left\{T_{l}(x)\right\}}$ may not converge to any limit.

It is clear that a Gaussian field is asymptotically Gaussian; however, as shown in Ref. 25, characterizing non-Gaussian fields that are HFG can be a difficult task even if the underlying field $T$ is a simple transformation (for instance, the square) of some Gaussian random function. Conditions for the HFG property to hold in some non-Gaussian circumstances are given in Ref. 25 by using group representations-yielding some interesting connection with random walks on hypergroups associated with the power spectrum of $T$. We stress that the possible existence of HFG behavior entails deep consequences on CMB data analysis. On the one hand, in fact, parameter estimation on CMB data is largely dominated by likelihood approaches; hence, an asymptotically Gaussian behavior would great simplify the implementation of optimal procedures. On the other hand, testing for non-Gaussianity is a key ingredient in the validation of the so-called inflationary scenarios, and the possible existence of HFG components for non-Gaussian models might set a theoretical limit to the investigation in this area.

\section{E. Purpose and plan}

Our purpose in this paper is to investigate the relationships between the HFG and HFE properties under an assumption of Gaussian subordination, that is, by considering fields $T$ that can be written as a deterministic function of some isotropic, real-valued Gaussian field. We will mainly focus on the case of polynomial subordinations, where the polynomials are of the Hermite type. Note also that Gaussian subordination is the favored framework for CMB modeling in a non-Gaussian setting (see, e.g., Refs. 4, 16, and 46).

Our main finding is that, despite their apparent independence, the HFG and HFE properties will turn out to be very close in a broad class of circumstances, suggesting that ergodicity (and hence the possibility to draw asymptotically justifiable statistical inferences) and asymptotic Gaussianity are very tightly related in a high-resolution setting. This may lead, we believe, to important characterizations of Gaussian random fields and to a better understanding of the conditions for the validity of statistical inference procedures based on observations drawn from a unique realization of a compactly supported random field, as in the spherical case.

The plan of this paper is as follows. In Sec. II we review some background material on Clebsch-Gordan coefficients to make the paper as self-contained as possible. In Sec. III we state and prove our main result, establishing necessary and sufficient conditions for ergodicity and Gaussianity and exploring the link between them. Indeed, these conditions turn out to be ex- 
tremely close so that in Sec. IV we can discuss more thoroughly a special case of practical relevance, namely, the quadratic case. Section $\mathrm{V}$ is devoted to further discussion and directions for further research.

\section{A REVIEW OF CLEBSCH-GORDAN COEFFICIENTS}

In this section, for the reader's convenience, we recall some basic facts about representation theory of compact groups and Clebsch-Gordan coefficients, which are widely used in the sequel of the paper. We refer the reader to standard textbooks (for instance, Refs. 42 and 43) for further details and any unexplained definition.

We recall first that a complete set of irreducible matrix representations for $\mathrm{SO}(3)$ (the group of rotations in $\left.\mathrm{R}^{3}\right)$ is provided by the so-called Wigner's matrices $D^{l}$ of dimensions $(2 l+1) \times(2 l$ +1 ) for $l=0,1,2, \ldots$-see Ref. 42 (Chap. 4) for an analytic expression. It follows from standard representation theory that we can exploit the family $\left\{D^{l}\right\}_{l=0,1,2, \ldots}$ to build alternative (reducible) representations, either by taking the tensor product family $\left\{D^{l_{1}} \otimes D^{l_{2}}\right\}_{l_{1}, l_{2}}$, or by considering direct sums $\left\{\oplus_{l=\left|l_{2}-l_{1}\right|}^{l_{2}+l_{1}} D^{l}\right\}_{l_{1}, l_{2}}$; these representations have dimensions $\left(2 l_{1}+1\right)\left(2 l_{2}+1\right) \times\left(2 l_{1}+1\right)\left(2 l_{2}+1\right)$ and are unitarily equivalent, whence there exists a unitary matrix $C_{l_{1} l_{2}}$ such that

$$
\left\{D^{l_{1}} \otimes D^{l_{2}}\right\}=C_{l_{1} l_{2}}\left\{\oplus_{l=\left|l_{2}-l_{1}\right|}^{l_{2}+l_{1}} D^{l}\right\} C_{l_{1} l_{2}}^{*}
$$

Here, $C_{l_{1} l_{2}}$ is a $\left\{\left(2 l_{1}+1\right)\left(2 l_{2}+1\right) \times\left(2 l_{1}+1\right)\left(2 l_{2}+1\right)\right\}$ block matrix with blocks $C_{l_{1}\left(m_{1}\right) l_{2}}^{l}$ of dimensions $\left(2 l_{2}+1\right) \times(2 l+1), m_{1}=-l_{1}, \ldots, l_{1}$. The elements of such a block are indexed by $m_{2}$ (over rows) and $m$ (over columns). More precisely,

$$
\begin{gathered}
C_{l_{1} l_{2}}=\left[C_{l_{1}\left(m_{1}\right) l_{2}}^{l \cdot} \cdot\right]_{m_{1}=-l_{1}, \ldots, l_{1} ; l=\left|l_{2}-l_{1}\right|, \ldots, l_{2}+l_{1}}, \\
C_{l_{1}\left(m_{1}\right) l_{2} .}^{l .}=\left\{C_{l_{1} m_{1} l_{2} m_{2}}^{l m}\right\}_{m_{2}=-l_{2}, \ldots, l_{2} ; m=-l, \ldots, l} .
\end{gathered}
$$

The Clebsch-Gordan coefficients for $\mathrm{SO}(3)$ are then defined as $\left\{C_{l_{1} m_{1} l_{2} m_{2}}^{l m}\right\}$, the elements of the unitary matrices $C_{l_{1} l_{2}}$. These coefficients are well known in the quantum theory of angular momentum, where $C_{l_{1} m_{1} l_{2} m_{2}}^{l_{1}}$ represents the probability amplitude that two quantum particles with total angular momenta $l_{1}$ and $l_{2}$ and momentum projections on the $z$-axis $m_{1}$ and $m_{2}$ are coupled to form a system with total angular momentum $l$ and projection $m$ (see, e.g., Ref. 22). Their use in the analysis of isotropic random fields is much more recent (see, for instance, Ref. 16 and references therein). Analytic expressions for the Clebsch-Gordan coefficients of $\mathrm{SO}(3)$ are known, but they are, in general, hardly manageable. We have, for instance [see Ref. 42, Expression (8.2.1.5)],

$$
\begin{aligned}
C_{l_{1} m_{1} l_{2} m_{2}}^{l_{3}-m_{3}}:= & (-1)^{l_{1}+l_{3}+m_{2}} \sqrt{2 l_{3}+1}\left[\frac{\left(l_{1}+l_{2}-l_{3}\right) !\left(l_{1}-l_{2}+l_{3}\right) !\left(l_{1}-l_{2}+l_{3}\right) !}{\left(l_{1}+l_{2}+l_{3}+1\right) !}\right]^{1 / 2} \\
& \times\left[\frac{\left(l_{3}+m_{3}\right) !\left(l_{3}-m_{3}\right) !}{\left(l_{1}+m_{1}\right) !\left(l_{1}-m_{1}\right) !\left(l_{2}+m_{2}\right) !\left(l_{2}-m_{2}\right) !}\right]^{1 / 2} \\
& \times \sum_{z} \frac{(-1)^{z}\left(l_{2}+l_{3}+m_{1}-z\right) !\left(l_{1}-m_{1}+z\right) !}{z !\left(l_{2}+l_{3}-l_{1}-z\right) !\left(l_{3}+m_{3}-z\right) !\left(l_{1}-l_{2}-m_{3}+z\right) !}
\end{aligned}
$$

where the summation runs over all z's such that the factorials are non-negative. This expression becomes somewhat neater for $m_{1}=m_{2}=m_{3}=0$, where we have 


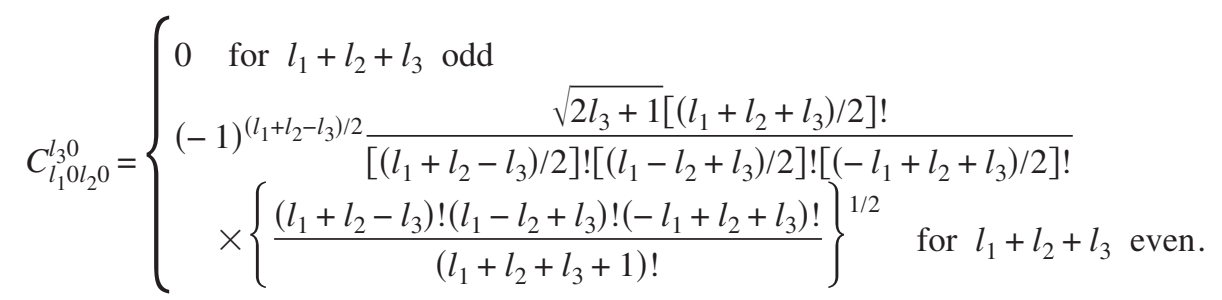

The coefficients enjoy also a nice set of symmetry and orthogonality properties, which will play a crucial role in our results to follow. For instance, from unitary equivalence, we deduce that

$$
\begin{gathered}
\sum_{m_{1}, m_{2}} C_{l_{1} m_{1} l_{2} m_{2}}^{l m} C_{l_{1} m_{1} l_{2} m_{2}}^{l^{\prime} m^{\prime}}=\delta_{l}^{l^{\prime}} \delta_{m}^{m^{\prime}}, \\
\sum_{l, m} C_{l_{1} m_{1} l_{2} m_{2}}^{l m} C_{l_{1} m_{1}^{\prime} l_{2} m_{2}^{\prime}}^{l m}=\delta_{m_{1}}^{m_{1}^{\prime}} \delta_{m_{2}}^{m_{2}^{\prime}} .
\end{gathered}
$$

Other properties are better expressed in terms of the Wigner $3 j$ coefficients, which are related to the Clebsch-Gordan by the identities (see Ref. 42, Chap. 8),

$$
\begin{gathered}
\left(\begin{array}{ccc}
l_{1} & l_{2} & l_{3} \\
m_{1} & m_{2} & m_{3}
\end{array}\right)=(-1)^{l_{3}+m_{3}} \frac{1}{\sqrt{2 l_{3}+1}} C_{l_{1}-m_{1} l_{2}-m_{2}}^{l_{3} m_{3}}, \\
C_{l_{1} m_{1} l_{2} m_{2}}^{l_{3} m_{3}}=(-1)^{l_{1}-l_{2}+m_{3}} \sqrt{2 l_{3}+1}\left(\begin{array}{ccc}
l_{1} & l_{2} & l_{3} \\
m_{1} & m_{2} & -m_{3}
\end{array}\right) .
\end{gathered}
$$

We have the following.

(a) The Wigner $3 j$ and the Clebsch-Gordan coefficients are real valued.

(b) (Triangle conditions) The Wigner $3 j$ and the Clebsch-Gordan coefficients are different from zero only if $m_{1}+m_{2}+m_{3}=0$ and $l_{i} \leq l_{j}+l_{k}$ for all $i, j, k=1,2,3$.

(c) (Parity) For any triple $l_{1}, l_{2}, l_{3}$,

$$
\left(\begin{array}{ccc}
l_{1} & l_{2} & l_{3} \\
m_{1} & m_{2} & m_{3}
\end{array}\right)=(-1)^{l_{1}+l_{2}+l_{3}}\left(\begin{array}{ccc}
l_{1} & l_{2} & l_{3} \\
-m_{1} & -m_{2} & -m_{3}
\end{array}\right) .
$$

(d) (Symmetry) For any triple $l_{1}, l_{2}, l_{3}$,

$$
\begin{aligned}
\left(\begin{array}{ccc}
l_{1} & l_{2} & l_{3} \\
m_{1} & m_{2} & m_{3}
\end{array}\right) & =\left(\begin{array}{ccc}
l_{2} & l_{3} & l_{1} \\
m_{2} & m_{3} & m_{1}
\end{array}\right)=\left(\begin{array}{ccc}
l_{3} & l_{1} & l_{2} \\
m_{3} & m_{1} & m_{2}
\end{array}\right)=(-1)^{l_{1}+l_{2}+l_{3}}\left(\begin{array}{ccc}
l_{3} & l_{2} & l_{1} \\
m_{3} & m_{2} & m_{1}
\end{array}\right) \\
& =(-1)^{l_{1}+l_{2}+l_{3}}\left(\begin{array}{ccc}
l_{1} & l_{3} & l_{2} \\
m_{1} & m_{3} & m_{2}
\end{array}\right)=(-1)^{l_{1}+l_{2}+l_{3}}\left(\begin{array}{ccc}
l_{2} & l_{1} & l_{3} \\
m_{2} & m_{1} & m_{3}
\end{array}\right) .
\end{aligned}
$$

\section{A GENERAL STATEMENT ABOUT GAUSSIAN SUBORDINATED FIELDS}

The two notations (1) and (2) are adopted throughout the sequel. Let us first recall a few basic facts and definitions.

(I) The first point concerns a characterization of isotropy in terms of angular power spectra. Indeed, as discussed in Refs. 16 and 27, if a random field is isotropic with finite fourthorder moment, then there exists necessarily an array $\left\{\mathcal{T}_{l_{1} l_{2}}^{3 l_{l}}(L)\right\}$ such that 


$$
\operatorname{cum}\left\{a_{l_{1} m_{1}}, a_{l_{2} m_{2}}, a_{l_{3} m_{3}}, a_{l_{4} m_{4}}\right\}=\sum_{L M}(-1)^{M}\left(\begin{array}{ccc}
l_{1} & l_{2} & L \\
m_{1} & m_{2} & M
\end{array}\right)\left(\begin{array}{ccc}
l_{3} & l_{4} & L \\
m_{3} & m_{4} & -M
\end{array}\right)(2 L+1) \mathcal{T}_{l_{1} l_{2}}^{l_{3} l_{4}}(L) .
$$

In general, the symbol $\operatorname{cum}\left\{X_{1}, \ldots, X_{m}\right\}$ denotes the joint cumulant of the random variables $X_{1}, \ldots, X_{m}$. Also, we label as usual $\left\{\mathcal{I}_{l_{1} l_{2}}^{t_{3} l_{4}}(L)\right\}$ the cumulant trispectrum of the random field (see, for instance, Refs. 16 and 27); as made clear by our notation, the quantity $\mathcal{T}_{l_{1} l_{2}}^{l_{3} l_{4}}(L)$ does not depend on $m_{1}, m_{2}, m_{3}$, and $m_{4}$ (this phenomenon is analogous to the fact that the power spectrum only depends on the frequency $l$-see Ref. 27 for a discussion of this point). As noted by $\mathrm{Hu},{ }^{16}$ geometrically the multipoles $\left(l_{1}, l_{2}, l_{3}, l_{4}\right)$ can be viewed as the sides of a quadrilateral, and $L$ as one of its main diagonals; $L$ is also the shared size of the two triangles formed by the corresponding pairs of sides. Clebsch-Gordan coefficients ensure that the triangle conditions are satisfied; indeed, they are different from zero only if $l_{1} \leq l_{2}+L, l_{2} \leq l_{1}+L$, and $L \leq l_{1}+l_{2}$.

(II) We shall sometimes label a point $x$ of the sphere $S^{2}$ in terms of its spherical coordinates, that is, $x=(\vartheta, \varphi)$, where $0 \leq \vartheta \leq \pi$ and $0 \leq \varphi<2 \pi$.

(III) Easy considerations yield the important fact that for any isotropic random field $T$,

$$
T_{l}(\vartheta, \varphi)=T_{l}(\bar{N})=\sum_{l m} a_{l m} Y_{l m}(\bar{N})=a_{l 0} \sqrt{\frac{2 l+1}{4 \pi}},
$$

where we denote by $\bar{N}:=(0,0)$ the North Pole of the sphere and by " $=$ " the equality in law between two random elements.

(IV) It is immediate that if $T$ is isotropic, then for every deterministic function $F$ the subordinated random application $x \mapsto F(T(x))$ is also isotropic. Moreover, if $F(T(x))$ is square integrable, then $F(T(\cdot))$ also admits a harmonic expansion analogous to (1). One specific instance of this situation is obtained by choosing $T$ to be Gaussian and isotropic, and $F$ to be any of the Hermite polynomials $\left\{H_{q}: q \geq 0\right\}$ (in this case, one talks about a Gaussian subordination of the Hermite type). We recall that the polynomials $H_{q}$ are such that $H_{q}$ $=\delta^{q} 1$, where 1 stands for the function which is constantly equal to $1, \delta^{0}$ is the identity, and $\delta^{q}(q \geq 1)$ represents the $q$ th iteration of the divergence operator $\delta$ acting on smooth functions as $\delta f(x)=x f(x)-f^{\prime}(x)$. For instance, $H_{0}=\mathbf{1}, H_{1}(x)=x, H_{2}(x)=x^{2}-1$, and so on. When $T$ is Gaussian, we adopt the notation

$$
H_{q}(T(x)):=T(x ; q)=\sum_{l=0}^{\infty} T_{l}(x ; q), \quad x \in S^{2}, \quad q \geq 2,
$$

where

$$
T_{l}(x ; q)=\sum_{m=-l}^{l} a_{l m ; q} Y_{l m}(x)
$$

is the $l$ th frequency component of $T(\cdot ; q)$, with $a_{l m ; q}$ as the associated harmonic coefficients. We shall also write $\left\{C_{l ; q}: l \geq 0\right\}$ and $\left\{\mathcal{T}_{l l}^{l l}(L ; q)\right\}$, respectively, for the power spectrum and for the cumulant trispectrum of $T(\cdot ; q)$, as introduced at point (I). According to Ref. 25 (Theorem 3), one has that $C_{l ; q}$ admits the following expansion in terms of the power spectrum $\left\{C_{l}\right\}$ of $T$ :

$$
C_{l ; q}=q ! \sum_{l_{1}, \ldots, l_{q}=0}^{\infty} C_{l_{1}} \cdots C_{l_{q}} \frac{4 \pi}{2 l+1}\left\{\prod_{i=1}^{q} \frac{2 l_{i}+1}{4 \pi}\right\} \sum_{L_{1}, \ldots, L_{q-2}}\left\{C_{l_{1}, 0 ; \ldots, l_{q}}^{L_{1}, L_{2}, \ldots, L_{q-2}, l ; 0}\right\}^{2},
$$

where $C_{l_{1}, 0, \ldots ; q_{q} L^{0}}^{L_{1}, L_{2}, \ldots, L_{q-2} ; 0}$ indicates a convolution of Clebsch-Gordan coefficients, that is, 


$$
C_{l_{1}, m_{1}, \ldots ; l_{p} m_{p}}^{\lambda_{1}, \lambda_{2}, \ldots, \lambda_{p-1} ; \mu}:=\sum_{\mu_{1}=-\lambda_{1}}^{\lambda_{1}} \cdots \sum_{\mu_{p-2}=-\lambda_{p-2}}^{\lambda_{p-2}} C_{l_{1}, m_{1}, l_{2}, m_{2}}^{\lambda_{1}, \mu_{1}} C_{\lambda_{1}, \mu_{1} ; l_{3}, m_{3}}^{\lambda_{2}, \mu_{2}} \cdots C_{\lambda_{p-2}, \mu_{p-2} ; l_{p}, m_{p}}^{\lambda_{p-1}, \mu}
$$

[see Refs. 25 and 27 for more details on these convolutions, which can also be viewed as probability amplitudes in alternative coupling schemes for quantum angular momenta (cf. Ref. 5)].

(V) An easy but important remark is the following. Since the expansion (1) is in order, the law of a centered isotropic Gaussian field $T$ is completely encoded by the power spectrum $\left\{C_{l}: l \geq 0\right\}$. This is a consequence of the fact that, in this case, the array $\left\{a_{l m}: l \geq 0, m\right.$ $=0, \ldots, l\}$ is composed of independent Gaussian random variables such that (i) $a_{l 0}$ is real valued and (ii) for every $m \geq 1$, the coefficient $a_{l m}$ has independent and equidistributed real and imaginary parts.

As anticipated, we shall now prove some new connections between HFE and HFG spherical fields (see Definitions 1 and 3), in the special case of fields of the type $T(\cdot ; q)$, as defined in (16). In particular, our main finding (as stated in Theorem 4) note that the conditions appearing in the following statement involve the coefficients $C_{l ; q}$ given in (18), and that these coefficients are completely determined by the power spectrum of the underlying Gaussian field $T$. For the result to follow, we shall need the notation

$$
w_{1 l}(L):=\left(C_{l 0 l 0}^{L 0}\right)^{2} \quad \text { and } \quad w_{2 l}(L):=\frac{(2 L+1)}{(2 l+1)^{2}}
$$

in such a way that

$$
\sum_{L=0}^{2 l} w_{1 l}(L)=\sum_{L=0}^{2 l} w_{2 l}(L)=1
$$

The fact that the weights $\left\{w_{1 l}(L)\right\}$ sum to one will be established during the proof of the theorem, while the analog identity for $\left\{w_{2 l}(L)\right\}$ is trivial.

Theorem 4: Let $q \geq 2$ and define $T(\cdot ; q)$ according to (16), where $T$ is Gaussian and isotropic. Let $\mathcal{T}_{l_{1} l_{2}}^{l_{2} l_{4}}(L ; q)$ be the reduced trispectrum of $T$. Then, the following holds.

(1) The random field $T(\cdot ; q)$ is $H F G$ if and only if

$$
\lim _{l \rightarrow \infty} \sum_{L=0}^{2 l} w_{1 l}(L) \frac{\mathcal{T}_{l l}^{l l}(L ; q)}{C_{l ; q}^{2}}=0 .
$$

(2) On the other hand, $T(\cdot ; q)$ is HFE if and only if

$$
\lim _{l \rightarrow \infty} \sum_{L=0}^{2 l} w_{2 l}(L) \frac{\mathcal{T}_{l l}^{l l}(L ; q)}{C_{l ; q}^{2}}=0
$$

Before proving Theorem 4, we shall note that $\left\{C_{l 0 l 0}^{L 0}\right\}^{2}$ is different from zero only for $L$ even, and $\mathcal{T}_{l l}^{l l}(L)$ is not, in general, positive valued. Moreover, in view of the forthcoming Lemma 5, also in (21) the sum runs only over even values of $L$.

Lemma 5: $\mathcal{T}_{l l}^{l l}(L)$ is zero when $L$ is odd.

Proof: From Ref. 16 [Eq. (17)], we infer that, in general,

$$
\mathcal{T}_{l_{1} l_{2}}^{t_{3} l_{4}}(L)=(-1)^{l_{1}+l_{2}+L} \mathcal{T}_{l_{1} l_{2}}^{t_{3} l_{4}}(L) .
$$

Considering the case $l_{1}=l_{2}=l_{3}=l_{4}=l$, we obtain the desired result.

Proof of Theorem 4: (Proof of 1) Consider the random spherical field 


$$
(\vartheta, \varphi) \mapsto \hat{T}_{l ; q}(\vartheta, \varphi):=\frac{T_{l ; q}(\vartheta, \varphi)}{\sqrt{\operatorname{Var}\left\{T_{l ; q}(\bar{N})\right\}}} \quad(\vartheta, \varphi) \in[0, \pi] \times[0,2 \pi),
$$

where $\bar{N}$ is the North Pole, and observe that, by isotropy and for every $(\vartheta, \varphi)$,

$$
\hat{T}_{l ; q}(\vartheta, \varphi)=\frac{a_{l 0}}{\sqrt{4 \pi C_{l ; q}}}
$$

The field $\hat{T}_{l ; q}$ is mean zero and has unit variance: since it also belongs to the $q$ th Wiener chaos associated with $T$, we can deduce from the results in Ref. 30 that it is asymptotically Gaussian if and only if

$$
\lim _{l \rightarrow \infty} \frac{1}{C_{l ; q}^{2}} \operatorname{cum}_{4}\left\{a_{l 0 ; q}\right\}=0 .
$$

As discussed, e.g., in Refs. 16 and 27, isotropy entails that we can write the fourth-order cumulant as

$$
\operatorname{cum}_{4}\left\{a_{l 0 ; q}\right\}=\sum_{L M}(-1)^{M}\left(\begin{array}{ccc}
l & l & L \\
0 & 0 & M
\end{array}\right)\left(\begin{array}{ccc}
l & l & L \\
0 & 0 & -M
\end{array}\right)(2 L+1) \mathcal{T}_{l l}^{l l}(L)=\sum_{L}\left(\begin{array}{ccc}
l & l & L \\
0 & 0 & 0
\end{array}\right)^{2}(2 L+1) \mathcal{T}_{l l}^{l l}(L)
$$

where the second equality follows because the corresponding Clebsch-Gordan coefficients are identically zero unless $M=0$ [see Sec. II, property (b)]. Hence, the field is asymptotically Gaussian if and only if

$$
\lim _{l \rightarrow \infty} \frac{1}{C_{l ; q}^{2}} \sum_{L}\left(\begin{array}{lll}
l & l & L \\
0 & 0 & 0
\end{array}\right)^{2}(2 L+1) \mathcal{T}_{l l}^{l l}(L)=0 .
$$

Since relation (14) is in order, we write

$$
\left(\begin{array}{lll}
l & l & L \\
0 & 0 & 0
\end{array}\right)^{2}(2 L+1)=\left\{C_{l 0 l 0}^{L 0}\right\}^{2}
$$

entailing, in turn, that

$$
\sum_{L}\left(\begin{array}{lll}
l & l & L \\
0 & 0 & 0
\end{array}\right)^{2}(2 L+1)=\sum_{L=0}^{2 l}\left\{C_{l 0 l 0}^{L 0}\right\}^{2}=\sum_{L=0}^{2 l} \sum_{M=-L}^{L}\left\{C_{l 0 l 0}^{L M}\right\}^{2} \equiv 1
$$

where the second equality follows from the fact that Clebsch-Gordan coefficients $C_{l_{1} m_{1} l_{2} m_{2}}^{l_{3} m_{3}}$ are different from zero only for $m_{3}=m_{1}+m_{2}$, and the third equality is a consequence from the orthonormality properties of the coefficients (which are the elements of unitary matrices whose rows are indexed by $m_{1}$ and $m_{2}$, and whose columns are indexed by $l_{3}$ and $m_{3}$ - see Sec. II for a review of these properties). We therefore have

$$
\frac{1}{C_{l ; q}^{2}} \operatorname{cum}_{4}\left\{a_{l 0 ; q}\right\}=\frac{1}{C_{l ; q}^{2}} \sum_{L}\left\{C_{l 0 l 0}^{L 0}\right\}^{2} \mathcal{T}_{l l}^{l l}(L)
$$

yielding the desired conclusion.

Proof of 2: On the other hand, we also obtain 


$$
\begin{aligned}
& E\left\{\frac{\hat{C}_{l ; q}}{C_{l ; q}}-1\right\}^{2}=\operatorname{Var}\left\{\frac{\hat{C}_{l ; q}}{C_{l ; q}}-1\right\} \\
& =\frac{1}{(2 l+1)^{2}} \frac{1}{C_{l ; q}^{2}} \sum_{m_{1} m_{2}} \operatorname{cum}\left\{a_{l m_{1} ; q}, \bar{a}_{l m_{1} ; q,} a_{l m_{2} ; q}, \bar{a}_{l m_{2} ; q}\right\}+\frac{2}{(2 l+1)^{2}} \frac{1}{C_{l ; q}^{2}} \sum_{m}\left\{E\left|a_{l m ; q}\right|^{2}\right\}^{2} \\
& =\frac{1}{(2 l+1)^{2}} \frac{1}{C_{l ; q}^{2}} \sum_{m_{1} m_{2}}(-1)^{m_{1}+m_{2}} \operatorname{cum}\left\{a_{l m_{1} ; q}, a_{l,-m_{1} ; q}, a_{l m_{2} ; q}, a_{l,-m_{2} ; q}\right\}+\frac{2}{(2 l+1)} \\
& =\frac{2}{(2 l+1)^{2}} \frac{1}{C_{l ; q}^{2}} \sum_{m_{1} m_{2}} \sum_{L M}(-1)^{M+m_{1}+m_{2}}\left(\begin{array}{ccc}
l & l & L \\
m_{1} & m_{2} & M
\end{array}\right)\left(\begin{array}{ccc}
l & l & L \\
-m_{1} & -m_{2} & -M
\end{array}\right)(2 L+1) \mathcal{T}_{l l}^{l l}(L)+\frac{2}{(2 l+1)} \\
& =\frac{2}{(2 l+1)^{2}} \frac{1}{C_{l ; q}^{2}} \sum_{\substack{L=0 \\
L \text { even }}}^{2 l}(2 L+1) \mathcal{T}_{l l}^{l l}(L)+\frac{2}{(2 l+1)} .
\end{aligned}
$$

It is obvious that $\Sigma_{L=0}^{2 l}(2 L+1)=(2 l+1)^{2}$, so that we have

$$
E\left\{\frac{\hat{C}_{l ; q}}{C_{l ; q}}-1\right\}^{2}=2 \sum_{\substack{L=0 \\ L \text { even }}}^{2 l} w_{l L}^{l l} \mathcal{T}_{l l}^{l l}(L)+\frac{2}{(2 l+1)}, \quad \text { where } w_{l L} \geq 0 \text { and } \sum_{\substack{L=0 \\ L \text { even }}}^{2 l} w_{l L}=1 .
$$

The result now follows immediately.

Remark: Note that

$$
\begin{gathered}
\left\{C_{l 0 l 0}^{L 0}\right\}^{2}=\frac{(2 L+1)\left(\frac{2 l+L}{2} !\right)^{2}}{\left(\frac{L}{2} !\right)^{2}} \frac{(L !)^{2}(2 l-L) !}{(2 l+L+1) !} \leq \frac{1}{(2 L+1)}, \\
w_{2 l}(L)=\frac{(2 L+1)}{(2 l+1)^{2}} \leq \frac{1}{2 l+1} .
\end{gathered}
$$

Note also that in the Gaussian case (e.g., $q=1$ ) we have $\mathcal{T}_{l l}^{t l}(L) \equiv 0$, whence

$$
E\left\{\frac{\hat{C}_{l ; q}}{C_{l ; q}}-1\right\}^{2}=\frac{2}{(2 l+1)} \rightarrow 0
$$

as expected.

The previous result strongly suggests that the conditions for asymptotic Gaussianity (HFG) and for ergodicity (HFE) should be tightly related. Indeed, we conjecture that HFE and HFG are equivalent in the case of Hermite-type Gaussian subordinations (and most probably even in more general circumstances). However, proving this claim seems analytically too demanding at this stage so for the rest of the paper we content ourselves with a detailed analysis of quadratic Gaussian subordinations. In particular, we believe that the content of Sec. IV (which is already quite technical) may provide the seed for a complete understanding of the HFG-HFE connection.

Remark 6: It should be noted that the reduced trispectrum satisfies [see Ref. 16, Eq. (16)] 


$$
\mathcal{T}_{l l}^{l l}\left(L^{\prime}\right)=\sum_{L}(2 L+1)\left\{\begin{array}{ccc}
l & l & L \\
l & l & L^{\prime}
\end{array}\right\} \mathcal{T}_{l l}^{l l}(L)
$$

In the previous remark, we introduced the well-known Wigner's $6 j$ coefficients, which intertwine alternative coupling schemes of three quantum angular momenta (see Refs. 5 and 42 for further properties and much more discussion). Their relationship with Wigner's $3 j$ coefficients is provided by the identity

$$
\left\{\begin{array}{lll}
a & b & e \\
c & d & f
\end{array}\right\}:=\sum_{\substack{\alpha, \beta, \gamma \\
\varepsilon, \delta, \phi}}(-1)^{e+f+\varepsilon+\phi}\left(\begin{array}{ccc}
a & b & e \\
\alpha & \beta & \varepsilon
\end{array}\right)\left(\begin{array}{ccc}
c & d & e \\
\gamma & \delta & -\varepsilon
\end{array}\right)\left(\begin{array}{ccc}
a & d & f \\
\alpha & \delta & -\phi
\end{array}\right)\left(\begin{array}{ccc}
c & b & f \\
\gamma & \beta & \phi
\end{array}\right)
$$

(see Ref. 42, Chap. 9, for analytic expressions and a full set of properties).

\section{THE QUADRATIC CASE}

\section{A. The class $\mathfrak{D}$ and main results}

As anticipated, the purpose of this section is to provide a more detailed and explicit analysis of the quadratic case $q=2$. For simplicity, in the sequel we consider a centered Gaussian isotropic spherical field $T$ such that $\operatorname{Var}(T(x))=\Sigma_{l}(2 l+1) C_{l} / 4 \pi=1$, where $\left\{C_{l}\right\}$ is as before the power spectrum of $T$. We start by recalling the notation

$$
T(x ; 2)=H_{2}(T(x))=\sum_{l_{1}, l_{2}=1}^{\infty} \sum_{m_{1} m_{2}} a_{l_{1} m_{1}} a_{l_{2} m_{2}} Y_{l_{1} m_{1}}(x) Y_{l_{2} m_{2}}(x)-1,
$$

where $T$ is isotropic, centered, and Gaussian. Our first result can be seen as a consequence of formula (18) (or, more generally, of the results in Ref. 25). Here, we provide a proof for the sake of completeness.

Lemma 7: The angular power spectrum of the squared random field (28) is given by

$$
C_{l ; 2}=E\left|a_{l m ; 2}\right|^{2}=2 \sum_{l_{1} l_{2}} C_{l_{1}} C_{l_{2}}\left(\begin{array}{ccc}
l_{1} & l_{2} & l \\
0 & 0 & 0
\end{array}\right)^{2} \frac{\left(2 l_{1}+1\right)\left(2 l_{2}+1\right)}{4 \pi} .
$$

Proof: Recall first that $Y_{00}(x) \equiv(4 \pi)^{-1 / 2}$ [see Ref. 42, Eq. (5.13.1.1)]. Hence, in view of (28), we have that, for $l=0$,

$$
\begin{aligned}
a_{00 ; 2} & =\int_{S^{2}}\left\{\sum_{l_{1} l_{2}} \sum_{m_{1} m_{2}} a_{l_{1} m_{1}} a_{l_{2} m_{2}} Y_{l_{1} m_{1}}(x) Y_{l_{2} m_{2}}(x)-1\right\} \bar{Y}_{00}(x) d x \\
& =\frac{1}{\sqrt{4 \pi}} \sum_{l_{1} l_{2}} \sum_{m_{1} m_{2}} a_{l_{1} m_{1}} \bar{a}_{l_{2} m_{2}}\left\{\int_{S^{2}} Y_{l_{1} m_{1}}(x) \bar{Y}_{l_{2} m_{2}}(x) d x\right\}-\sqrt{4 \pi} \\
& =\frac{1}{\sqrt{4 \pi}} \sum_{l_{1} l_{2}} \sum_{m_{1} m_{2}} a_{l_{1} m_{1}} \bar{a}_{l_{2} m_{2}} \delta_{l_{1}}^{2} \delta_{m_{1}}^{m_{2}}-\sqrt{4 \pi} \\
& =\frac{1}{\sqrt{4 \pi}} \sum_{l m}\left|a_{l m}\right|^{2}-\sqrt{4 \pi} .
\end{aligned}
$$

It follows that

$$
E a_{00 ; 2}=\sum_{l} \frac{2 l+1}{\sqrt{4 \pi}} C_{l}-\sqrt{4 \pi}=\sqrt{4 \pi}\left\{\sum_{l} \frac{2 l+1}{4 \pi} C_{l}-1\right\}=0
$$

and 


$$
E H_{2}(T(x))=E \sum_{l=0}^{\infty} a_{l m ; 2} Y_{l m}(x)=E a_{00 ; 2} Y_{00}(x)=0,
$$

the second step follows because $E a_{l m}=0$ for all $l>0$ under isotropy (see Ref. 2). Indeed, we have [from (28) and in view of (17)]

$$
\begin{aligned}
a_{l m ; 2} & =\int_{S^{2}} \sum_{l_{1} l_{2}} \sum_{m_{1} m_{2}} a_{l_{1} m_{1}} a_{l_{2} m_{2}} Y_{l m_{1}}(x) Y_{l m_{2}}(x) \bar{Y}_{l m}(x) d x \\
& =\sum_{l_{1}, l_{2}=1}^{\infty} \sum_{m_{1} m_{2}} a_{l_{1} m_{1}} a_{l_{2} m_{2}}\left(\begin{array}{ccc}
l_{1} & l_{2} & l \\
m_{1} & m_{2} & -m
\end{array}\right)\left(\begin{array}{ccc}
l_{1} & l_{2} & l \\
0 & 0 & 0
\end{array}\right) \sqrt{\frac{\left(2 l_{1}+1\right)\left(2 l_{2}+1\right)(2 l+1)}{4 \pi}} .
\end{aligned}
$$

Note that the constant term -1 has no effect for $l \geq 1$ because

$$
\int_{S^{2}} Y_{l m}(x) d x=0 \quad \text { for all } l \geq 1
$$

Now

$$
\begin{aligned}
& E\left|a_{l m ; 2}\right|^{2}=E\left\{\sum_{l_{1} l_{2}} \sum_{m_{1} m_{2}} a_{l_{1} m_{1}} a_{l_{2} m_{2}}\left(\begin{array}{ccc}
l_{1} & l_{2} & l \\
m_{1} & m_{2} & -m
\end{array}\right)\left(\begin{array}{ccc}
l_{1} & l_{2} & l \\
0 & 0 & 0
\end{array}\right) \sqrt{\frac{\left(2 l_{1}+1\right)\left(2 l_{2}+1\right)(2 l+1)}{4 \pi}}\right. \\
& \left.\times \sum_{l_{1}^{\prime} l_{2}^{\prime} m_{1}^{\prime} m_{2}^{\prime}} \bar{a}_{l_{1}^{\prime} m_{1}^{\prime}} \bar{a}_{l_{2}^{\prime} m_{2}^{\prime}}\left(\begin{array}{ccc}
l_{1}^{\prime} & l_{2}^{\prime} & l \\
m_{1}^{\prime} & m_{2}^{\prime} & -m
\end{array}\right)\left(\begin{array}{ccc}
l_{1}^{\prime} & l_{2}^{\prime} & l \\
0 & 0 & 0
\end{array}\right) \sqrt{\frac{\left(2 l_{1}^{\prime}+1\right)\left(2 l_{2}^{\prime}+1\right)(2 l+1)}{4 \pi}}\right\} \\
& =2 \sum_{l_{1} l_{2}} C_{l_{1}} C_{l_{2}} \sum_{m_{1} m_{2}}\left(\begin{array}{ccc}
l_{1} & l_{2} & l \\
m_{1} & m_{2} & -m
\end{array}\right)^{2}\left(\begin{array}{ccc}
l_{1} & l_{2} & l \\
0 & 0 & 0
\end{array}\right)^{2} \frac{\left(2 l_{1}+1\right)\left(2 l_{2}+1\right)(2 l+1)}{4 \pi} \\
& =2 \sum_{l_{1} l_{2}} C_{l_{1}} C_{l_{2}}\left(\begin{array}{ccc}
l_{1} & l_{2} & l \\
0 & 0 & 0
\end{array}\right)^{2} \frac{\left(2 l_{1}+1\right)\left(2 l_{2}+1\right)}{4 \pi},
\end{aligned}
$$

and the proof is completed.

Remark: Note that

$$
\begin{aligned}
\operatorname{Var}\left\{T^{2}(x)\right\} & =\sum_{l} \frac{2 l+1}{4 \pi} C_{l ; 2}=2 \sum_{l_{1} l_{2}} C_{l_{1}} C_{l_{2}} \frac{\left(2 l_{1}+1\right)\left(2 l_{2}+1\right)}{4 \pi}\left\{\sum_{l} \frac{2 l+1}{4 \pi}\left(\begin{array}{ccc}
l_{1} & l_{2} & l \\
0 & 0 & 0
\end{array}\right)^{2}\right\} \\
& =2 \sum_{l_{1} l_{2}} C_{l_{1}} C_{l_{2}} \frac{\left(2 l_{1}+1\right)\left(2 l_{2}+1\right)}{(4 \pi)^{2}}=2[\operatorname{Var}\{T(x)\}]^{2},
\end{aligned}
$$

as expected from standard property of Gaussian variables. Here, we have used again

$$
\sum_{l}(2 l+1)\left(\begin{array}{ccc}
l_{1} & l_{2} & l \\
0 & 0 & 0
\end{array}\right)^{2} \equiv 1 .
$$

Our strategy is now as follows. We shall first define a very general class, noted as $\mathfrak{D}$, of quadratic models in terms of the power spectrum of the underlying Gaussian field, and then we shall show that the two notions of HFG and HFE coincide within $\mathfrak{D}$.

Definition 8: The centered Gaussian isotropic field $T$ is said to belong to the class $\mathfrak{D}$ if there exist real numbers $\alpha$ and $\beta$ such that

(1) $\alpha \in \mathbb{R}$ and $\beta \geq 0$,

(2) $\sum_{l=0}^{\infty} l^{-\alpha+1} e^{-\beta l}<\infty$, and

(3) there exists constants $c_{1}, c_{2}>0$ such that 


$$
0<c_{1} \leq \lim _{l \rightarrow \infty} \frac{C_{l}}{l^{-\alpha} e^{-\beta l}} \leq \lim _{l \rightarrow \infty} \frac{C_{l}}{l^{-\alpha} e^{-\beta l}} \leq c_{2}<\infty .
$$

\section{Remarks:}

(1) As a first approximation, the class $\mathfrak{D}$ contains virtually all models that are relevant for CMB modeling in the case of a quadratic Gaussian subordination. For instance, Sachs-Wolfe models with the so-called Bardeen's potential entail a polynomial decay of the $C_{l}(\beta=0)$, whereas the so-called Silk damping effect entails an exponential decay of the power spectrum of primary $\mathrm{CMB}$ anisotropies at higher $l$. We refer again to textbooks such as Ref. 12 for more discussion on these points.

(2) Note that condition (2) in the definition of $\mathfrak{D}$ implies that the parameters $\alpha$ and $\beta$ must be such that either $\beta=0$ and $\alpha>2$, or $\beta>0$ and $\alpha \in \mathbb{R}$ (with no restrictions).

The next statement is the main achievement of this section. It shows, in particular, that the HFG and HFE exhibit the same phase transition within the class $\mathfrak{D}$.

Theorem 9: Let $T(\cdot ; 2)=H_{2}(T)$, where the centered Gaussian isotropic field $T(\cdot)$ is an element of the class $\mathfrak{D}$. Then, the following three conditions are equivalent:

(i) $T(\cdot ; 2)$ is $H F G$,

(ii) $T(\cdot ; 2)$ is $H F E$, and

(iii) $\beta>0$ and $\alpha \in \mathbb{R}$.

\section{B. Proof of Theorem 9}

From Ref. 25 (Sec. VI), we already know that conditions (i) and (iii) in the statement of Theorem 9 are equivalent. The proof of the remaining implication (ii) $\Leftrightarrow$ (iii) is divided into several steps.

We start by showing that if (iii) is not verified, then the angular power spectrum of the transformed field, under broad conditions, exhibits the same behavior as the angular power spectrum of the subordinating field.

Lemma 10: Suppose $\beta=0$ and $\alpha>2$, then

$$
\frac{3 \times 2^{\alpha}}{4 \pi} C_{l} \frac{c_{1}^{2}}{c_{2}} \leq C_{l ; 2} \leq \frac{c_{2}^{2}}{c_{1} \pi}\{2 \zeta(\alpha-1)+\zeta(\alpha)\} C_{l / 2}=O\left(C_{l}\right),
$$

where $\zeta(\cdot)$ denotes the Riemann zeta function.

Proof: We have

$$
\begin{aligned}
\sum_{l_{1} l_{2}} C_{l_{1}} C_{l_{2}}\left(\begin{array}{ccc}
l_{1} & l_{2} & l \\
0 & 0 & 0
\end{array}\right)^{2} \frac{\left(2 l_{1}+1\right)\left(2 l_{2}+1\right)}{4 \pi} & \leq 2 \sum_{l_{1} \leq l_{2}} C_{l_{1}} C_{l_{2}}\left(\begin{array}{ccc}
l_{1} & l_{2} & l \\
0 & 0 & 0
\end{array}\right)^{2} \frac{\left(2 l_{1}+1\right)\left(2 l_{2}+1\right)}{4 \pi} \\
& \leq 2 \frac{c_{2}}{c_{1}} C_{l / 2} \sum_{l_{1} \leq l_{2}} C_{l_{1}}\left(\begin{array}{lll}
l_{1} & l_{2} & l \\
0 & 0 & 0
\end{array}\right)^{2} \frac{\left(2 l_{1}+1\right)\left(2 l_{2}+1\right)}{4 \pi}
\end{aligned}
$$

because $\left(l_{1} \vee l_{2}\right)>l / 2$ by the triangle conditions and $\sup _{l_{2} \geq l / 2} C_{l_{2}} / C_{l / 2} \leq c_{2} / c_{1}$. Now

$$
\begin{aligned}
\sum_{l_{1} \leq l_{2}} C_{l_{1}}\left(\begin{array}{ccc}
l_{1} & l_{2} & l \\
0 & 0 & 0
\end{array}\right)^{2} \frac{\left(2 l_{1}+1\right)\left(2 l_{2}+1\right)}{4 \pi} & \leq \sum_{l_{1}} C_{l_{1}} \frac{\left(2 l_{1}+1\right)}{4 \pi} \sum_{l_{2}}\left(2 l_{2}+1\right)\left(\begin{array}{ccc}
l_{1} & l_{2} & l \\
0 & 0 & 0
\end{array}\right)^{2}=\sum_{l_{1}} C_{l_{1}} \frac{\left(2 l_{1}+1\right)}{4 \pi} \\
& <\infty .
\end{aligned}
$$

More precisely, 


$$
\sum_{l_{1}} C_{l_{1}} \frac{\left(2 l_{1}+1\right)}{4 \pi} \leq \frac{c_{2}}{4 \pi} \sum_{l}(2 l+1) l^{-\alpha} \leq \frac{c_{2}}{4 \pi}\{2 \zeta(\alpha-1)+\zeta(\alpha)\}
$$

Hence,

$$
C_{l ; 2} \leq \frac{c_{2}^{2}}{2 c_{1} \pi}\{2 \zeta(\alpha-1)+\zeta(\alpha)\} C_{l / 2}
$$

The upper bound is then established. For the lower bound, it is sufficient to show that

$$
\begin{aligned}
\sum_{l_{1} l_{2}} C_{l_{1}} C_{l_{2}}\left(\begin{array}{ccc}
l_{1} & l_{2} & l \\
0 & 0 & 0
\end{array}\right)^{2} \frac{\left(2 l_{1}+1\right)\left(2 l_{2}+1\right)}{4 \pi} \geq & \sum_{l_{2}} C_{1} C_{l_{2}}\left(\begin{array}{ccc}
l_{1} & l_{2} & l \\
0 & 0 & 0
\end{array}\right)^{2} \frac{3\left(2 l_{2}+1\right)}{4 \pi} \geq 3 \\
& \times 2^{\alpha} C_{l} \frac{c_{1}^{2}}{c_{2}} \sum_{l_{2}}\left(\begin{array}{lll}
l_{1} & l_{2} & l \\
0 & 0 & 0
\end{array}\right)^{2} \frac{\left(2 l_{2}+1\right)}{4 \pi} \geq \frac{3 \times 2^{\alpha}}{4 \pi} C_{l} \frac{c_{1}^{2}}{c_{2}},
\end{aligned}
$$

as claimed.

Loosely, Lemma 10 states that, under algebraic decay, the rate of convergence to zero of the angular power spectrum is not affected by a quadratic transformation, i.e., $C_{l ; 2} \simeq C_{l}$. The following result holds for fixed $l$, and it is therefore not related to the high-frequency asymptotic behavior of the power spectrum $\left\{C_{l}\right\}$ (see Ref. 25 for related computations). Note that we use the notation

$$
\hat{C}_{l ; 2}=\frac{1}{2 l+1} \sum_{m=-l}^{l}\left|a_{l m ; 2}\right|^{2}, \quad \widetilde{C}_{l ; 2}=\frac{\hat{C}_{l ; 2}}{C_{l ; 2}}
$$

Lemma 11: Let $T(. ; 2)$ be defined by (28). Then, we have

$$
E\left\{\widetilde{C}_{l ; 2}-1\right\}^{2}=\frac{16}{C_{l ; 2}^{2}} \sum_{l_{1} l_{2} l_{3}} C_{l_{1}}^{2} C_{l_{2}} C_{l_{3}}\left(\begin{array}{ccc}
l_{1} & l_{2} & l \\
0 & 0 & 0
\end{array}\right)^{2}\left(\begin{array}{ccc}
l_{1} & l_{3} & l \\
0 & 0 & 0
\end{array}\right)^{2} \frac{\left(2 l_{1}+1\right)\left(2 l_{2}+1\right)\left(2 l_{3}+1\right)}{(4 \pi)^{2}}+R(l),
$$

where for all $l=1,2, \ldots$,

$$
0 \leq R(l) \leq \frac{4}{2 l+1}
$$

Proof: In the sequel, we shall use repeatedly the unitary properties of Clebsch-Gordan coefficients, i.e.,

$$
\sum_{m_{1} m_{2}}\left(\begin{array}{ccc}
l & l & L \\
m_{1} & m_{2} & M
\end{array}\right)\left(\begin{array}{ccc}
l & l & L^{\prime} \\
m_{1} & m_{2} & M^{\prime}
\end{array}\right)=\frac{\delta_{L}^{L^{\prime}} \delta_{M}^{M^{\prime}}}{2 L+1}
$$

Recalling (23) and (24), we need to evaluate

$\frac{1}{(2 l+1)^{2} C_{l ; 2}^{2}} \sum_{m_{1} m_{2}} \operatorname{cum}\left\{a_{l m_{1}}, \bar{a}_{l m_{1}}, a_{l m_{2}}, \bar{a}_{l m_{2}}\right\}=\frac{1}{(2 l+1)^{2} C_{l ; 2}^{2}} \sum_{m_{1} m_{2}}(-1)^{m_{1}+m_{2}} \operatorname{cum}\left\{a_{l m_{1}}, a_{l,-m_{1}}, a_{l m_{2}}, a_{l,-m_{2}}\right\}$.

Now 


$$
\begin{aligned}
& \operatorname{cum}\left\{a_{l m_{1}}, a_{l,-m_{1}}, a_{l m_{2}}, a_{l,-m_{2}}\right\}=\operatorname{cum}\left\{\sum_{l_{1} l_{2}} \sum_{\mu_{1} \mu_{2}} a_{l_{1} \mu_{1}} a_{l_{2} \mu_{2}}\left(\begin{array}{ccc}
l_{1} & l_{2} & l \\
\mu_{1} & \mu_{2} & m_{1}
\end{array}\right)\right. \\
& \times\left(\begin{array}{ccc}
l_{1} & l_{2} & l \\
0 & 0 & 0
\end{array}\right) \sqrt{\frac{\left(2 l_{1}+1\right)\left(2 l_{2}+1\right)(2 l+1)}{4 \pi}}, \\
& \sum_{l_{3} l_{4}} \sum_{\mu_{3} \mu_{4}} a_{l_{3} \mu_{3}} a_{l_{4} \mu_{4}}\left(\begin{array}{ccc}
l_{3} & l_{4} & l \\
\mu_{3} & \mu_{4} & -m_{1}
\end{array}\right) \\
& \times\left(\begin{array}{ccc}
l_{3} & l_{4} & l \\
0 & 0 & 0
\end{array}\right) \sqrt{\frac{\left(2 l_{3}+1\right)\left(2 l_{4}+1\right)(2 l+1)}{4 \pi}}, \\
& \sum_{l_{5} l_{6}} \sum_{\mu_{5} \mu_{6}} a_{l \mu_{5}} a_{l \mu_{6}}\left(\begin{array}{ccc}
l_{5} & l_{6} & l \\
\mu_{5} & \mu_{6} & m_{2}
\end{array}\right) \\
& \times\left(\begin{array}{ccc}
l_{5} & l_{6} & l \\
0 & 0 & 0
\end{array}\right) \sqrt{\frac{\left(2 l_{5}+1\right)\left(2 l_{6}+1\right)(2 l+1)}{4 \pi}}, \\
& \sum_{l_{7} l_{8}} \sum_{\mu_{7} \mu_{8}} a_{l \mu_{7}} a_{l \mu_{8}}\left(\begin{array}{ccc}
l_{7} & l_{8} & l \\
\mu_{7} & \mu_{8} & -m_{2}
\end{array}\right) \\
& \left.\times\left(\begin{array}{ccc}
l_{7} & l_{8} & l \\
0 & 0 & 0
\end{array}\right) \sqrt{\frac{\left(2 l_{7}+1\right)\left(2 l_{8}+1\right)(2 l+1)}{4 \pi}}\right\},
\end{aligned}
$$

and counting equivalent permutations

$$
\begin{aligned}
& =8 \sum_{l_{1} l_{2} l_{3} l_{4}} \sum_{\mu_{1} \mu_{2} \mu_{3} \mu_{4}}(-1)^{\mu_{1}+\mu_{2}+\mu_{3}+\mu_{4}} C_{l_{1}} C_{l_{2}} C_{l_{3}} C_{l_{4}}\left(\begin{array}{ccc}
l_{1} & l_{2} & l \\
\mu_{1} & \mu_{2} & m_{1}
\end{array}\right) \\
& \times\left(\begin{array}{ccc}
l_{1} & l_{2} & l \\
0 & 0 & 0
\end{array}\right) \frac{(2 l+1)^{2} \Pi_{i=1}^{4}\left(2 l_{i}+1\right)}{(4 \pi)^{2}}\left(\begin{array}{ccc}
l_{1} & l_{3} & l \\
-\mu_{1} & -\mu_{3} & -m_{1}
\end{array}\right)\left(\begin{array}{ccc}
l_{1} & l_{3} & l \\
0 & 0 & 0
\end{array}\right)\left(\begin{array}{ccc}
l_{4} & l_{3} & l \\
\mu_{4} & \mu_{3} & m_{2}
\end{array}\right)\left(\begin{array}{ccc}
l_{4} & l_{3} & l \\
0 & 0 & 0
\end{array}\right) \\
& \times\left(\begin{array}{ccc}
l_{4} & l_{2} & l \\
-\mu_{4} & -\mu_{2} & m_{2}
\end{array}\right)\left(\begin{array}{ccc}
l_{4} & l_{2} & l \\
0 & 0 & 0
\end{array}\right)+8 \sum_{l_{1} l_{2} l_{3} l_{4}} \sum_{\mu_{1} \mu_{2} \mu_{3} \mu_{4}}(-1)^{\mu_{1}+\mu_{2}+\mu_{3}+\mu_{4}} C_{l_{1}} C_{l_{2}} C_{l_{3}} C_{l_{4}}\left(\begin{array}{ccc}
l_{1} & l_{2} & l \\
\mu_{1} & \mu_{2} & m_{1}
\end{array}\right) \\
& \times\left(\begin{array}{ccc}
l_{1} & l_{2} & l \\
0 & 0 & 0
\end{array}\right) \frac{(2 l+1)^{2} \Pi_{i=1}^{4}\left(2 l_{i}+1\right)}{(4 \pi)^{2}}\left(\begin{array}{ccc}
l_{3} & l_{4} & l \\
\mu_{3} & \mu_{4} & -m_{1}
\end{array}\right)\left(\begin{array}{ccc}
l_{3} & l_{4} & l \\
0 & 0 & 0
\end{array}\right)\left(\begin{array}{ccc}
l_{1} & l_{3} & l \\
-\mu_{1} & -\mu_{3} & m_{2}
\end{array}\right)\left(\begin{array}{ccc}
l_{1} & l_{3} & l \\
0 & 0 & 0
\end{array}\right) \\
& \times\left(\begin{array}{ccc}
l_{4} & l_{2} & l \\
-\mu_{4} & -\mu_{2} & -m_{2}
\end{array}\right)\left(\begin{array}{ccc}
l_{4} & l_{2} & l \\
0 & 0 & 0
\end{array}\right)+8 \\
& \times \sum_{l_{1} l_{2} l_{3} l_{4}} \sum_{\mu_{1} \mu_{2} \mu_{3} \mu_{4}}(-1)^{\mu_{1}+\mu_{2}+\mu_{3}+\mu_{4}} C_{l_{1}} C_{l_{2}} C_{l_{3}} C_{l_{4}}\left(\begin{array}{ccc}
l_{1} & l_{2} & l \\
\mu_{1} & \mu_{2} & m_{1}
\end{array}\right)\left(\begin{array}{ccc}
l_{1} & l_{2} & l \\
0 & 0 & 0
\end{array}\right)\left(\begin{array}{ccc}
l_{1} & l_{3} & l \\
-\mu_{1} & \mu_{3} & -m_{1}
\end{array}\right) \\
& \times\left(\begin{array}{lll}
l_{1} & l_{3} & l \\
0 & 0 & 0
\end{array}\right)\left(\begin{array}{ccc}
l_{2} & l_{4} & l \\
-\mu_{2} & \mu_{4} & m_{2}
\end{array}\right)\left(\begin{array}{ccc}
l_{2} & l_{4} & l \\
0 & 0 & 0
\end{array}\right)\left(\begin{array}{ccc}
l_{3} & l_{4} & l \\
-\mu_{3} & -\mu_{4} & -m_{2}
\end{array}\right)\left(\begin{array}{ccc}
l_{4} & l_{2} & l \\
0 & 0 & 0
\end{array}\right) \frac{(2 l+1)^{2} \Pi_{i=1}^{4}\left(2 l_{i}+1\right)}{(4 \pi)^{2}} \\
& =: 8\left\{A\left(m_{1},-m_{1}, m_{2},-m_{2}\right)+B\left(m_{1},-m_{1}, m_{2},-m_{2}\right)+C\left(m_{1},-m_{1}, m_{2},-m_{2}\right)\right\} \text {. }
\end{aligned}
$$

For the first term, note first that $(-1)^{m_{1}+m_{2}+\mu_{1}+\mu_{2}+\mu_{3}+\mu_{4}} \equiv 1$ because the exponent is necessarily even by the properties of Wigner's coefficients. Moreover, applying iteratively (31), 


$$
\begin{array}{rl}
\sum_{m_{1} m_{2}} & A\left(m_{1},-m_{1}, m_{2},-m_{2}\right) \\
= & \sum_{l_{1} l_{2} l_{3} l_{4}} \sum_{m_{2} \mu_{2}} \sum_{\mu_{3} \mu_{4}} C_{l_{1}} C_{l_{2}} C_{l_{3}} C_{l_{4}}\left(\begin{array}{lll}
l_{1} & l_{2} & l \\
0 & 0 & 0
\end{array}\right)\left(\begin{array}{lll}
l_{1} & l_{3} & l \\
0 & 0 & 0
\end{array}\right) \\
& \times \frac{(2 l+1)^{2} \Pi_{i=1}^{4}\left(2 l_{i}+1\right)}{(4 \pi)^{2}}\left(\begin{array}{ccc}
l_{4} & l_{3} & l \\
\mu_{4} & \mu_{3} & m_{2}
\end{array}\right)\left(\begin{array}{ccc}
l_{4} & l_{3} & l \\
0 & 0 & 0
\end{array}\right)\left(\begin{array}{ccc}
l_{4} & l_{2} & l \\
-\mu_{4} & -\mu_{2} & m_{2}
\end{array}\right)\left(\begin{array}{lll}
l_{4} & l_{2} & l \\
0 & 0 & 0
\end{array}\right) \frac{\delta_{\mu_{3}}^{\mu_{2}} \delta_{l_{2}}^{j_{3}}}{2 l_{3}+1} \\
= & \sum_{l_{1} l_{2} l_{3} l_{4}} C_{l_{1}} C_{l_{2}}^{2} C_{l_{4}}\left(\begin{array}{lll}
l_{1} & l_{2} & l \\
0 & 0 & 0
\end{array}\right)^{2}\left(\begin{array}{ccc}
l_{4} & l_{2} & l \\
0 & 0 & 0
\end{array}\right)^{2} \frac{\left(2 l_{1}+1\right)\left(2 l_{2}+1\right)\left(2 l_{4}+1\right)(2 l+1)^{2}}{(4 \pi)^{2}} .
\end{array}
$$

Likewise, for the second term we note that $(-1)^{\mu_{1}+\mu_{2}+\mu_{3}+\mu_{4}} \equiv 1$, and using (27),

$$
\begin{aligned}
\sum_{m_{1} m_{2}}(-1)^{m_{1}+m_{2}} B\left(m_{1},-m_{1}, m_{2},-m_{2}\right)= & \sum_{l_{1} l_{2} l_{3} l_{4}} C_{l_{1}} C_{l_{2}} C_{l_{3}} C_{l_{4}}\left\{\begin{array}{lll}
l_{1} & l_{3} & l \\
l_{4} & l_{2} & l
\end{array}\right\}\left(\begin{array}{ccc}
l_{1} & l_{2} & l \\
0 & 0 & 0
\end{array}\right)\left(\begin{array}{ccc}
l_{3} & l_{4} & l \\
0 & 0 & 0
\end{array}\right) \\
& \times\left(\begin{array}{ccc}
l_{1} & l_{3} & l \\
0 & 0 & 0
\end{array}\right)\left(\begin{array}{ccc}
l_{4} & l_{2} & l \\
0 & 0 & 0
\end{array}\right) \frac{(2 l+1)^{2} \Pi_{i=1}^{4}\left(2 l_{i}+1\right)}{(4 \pi)^{2}}
\end{aligned}
$$

Now by Cauchy-Schwartz inequality and recalling that

$$
\left|\left\{\begin{array}{lll}
l_{1} & l_{3} & l \\
l_{2} & l_{4} & l
\end{array}\right\}\right| \leq \frac{1}{2 l+1} \text { for all } l_{1}, l_{2}, l_{3}, l_{4},
$$

the previous quantity can be bounded by

$$
\begin{aligned}
\frac{1}{2 l+} & \sum_{l_{1} l_{2} l_{3} l_{4}} C_{l_{1}} C_{l_{2}} C_{l_{3}} C_{l_{4}}\left(\begin{array}{lll}
l_{1} & l_{2} & l \\
0 & 0 & 0
\end{array}\right)\left(\begin{array}{ccc}
l_{3} & l_{4} & l \\
0 & 0 & 0
\end{array}\right)\left(\begin{array}{lll}
l_{1} & l_{3} & l \\
0 & 0 & 0
\end{array}\right)\left(\begin{array}{lll}
l_{4} & l_{2} & l \\
0 & 0 & 0
\end{array}\right) \frac{(2 l+1)^{2} \Pi_{i=1}^{4}\left(2 l_{i}+1\right)}{(4 \pi)^{2}} \\
\leq & {\left[\sum_{l_{1} l_{2} l_{3} l_{4}} C_{l_{1}} C_{l_{2}} C_{l_{3}} C_{l_{4}}\left(\begin{array}{lll}
l_{1} & l_{2} & l \\
0 & 0 & 0
\end{array}\right)^{2}\left(\begin{array}{lll}
l_{3} & l_{4} & l \\
0 & 0 & 0
\end{array}\right)^{2} \frac{\left(2 l_{3}+1\right)\left(2 l_{4}+1\right)}{4 \pi} \frac{\left(2 l_{1}+1\right)\left(2 l_{2}+1\right)(2 l+1)}{4 \pi}\right]^{1 / 2} } \\
& \times\left[\sum_{l_{1} l_{2} l_{3} l_{4}} C_{l_{1}} C_{l_{2}} C_{l_{3}} C_{l_{4}}\left(\begin{array}{lll}
l_{1} & l_{3} & l \\
0 & 0 & 0
\end{array}\right)^{2}\left(\begin{array}{lll}
l_{2} & l_{4} & l \\
0 & 0 & 0
\end{array}\right)^{2}\right. \\
& \left.\times \frac{\left(2 l_{3}+1\right)\left(2 l_{4}+1\right)}{4 \pi} \frac{\left(2 l_{1}+1\right)\left(2 l_{2}+1\right)(2 l+1)}{4 \pi}\right]^{1 / 2}=\frac{2 l+1}{4} C_{l ; 2}^{2},
\end{aligned}
$$

whence

$$
\left|\frac{8}{(2 l+1)^{2} C_{l ; 2}^{2}} \sum_{m_{1}, m_{2}} B\left(m_{1},-m_{1}, m_{2},-m_{2}\right)\right| \leq \frac{2}{2 l+1} .
$$

It is easy to see that $\Sigma_{m_{1} m_{2}} A\left(m_{1},-m_{1}, m_{2},-m_{2}\right)=\Sigma_{m_{1} m_{2}} C\left(m_{1},-m_{1}, m_{2},-m_{2}\right)$. In view of (23) and (24), the statement of the lemma follows easily.

The proof of Theorem 9 is now concluded by the following lemma.

Lemma 12: If $\beta=0$ and $\alpha>2$, then

$$
\lim \inf _{l \rightarrow \infty} E\left\{\widetilde{C}_{l ; 2}-1\right\}^{2} \geq C_{2}^{2}\left\{\frac{c_{2}^{3}}{2 c_{1}^{2} \pi}\{2 \zeta(\alpha-1)+\zeta(\alpha)\} 2^{\alpha}\right\}^{2}>0 .
$$

If $\beta>0$ and $\alpha$ is real, then

$$
\lim _{l \rightarrow \infty} E\left\{\widetilde{C}_{l ; 2}-1\right\}^{2}=0
$$


Proof: For the first part, from Lemma 11 we can focus on

$$
\begin{aligned}
& \frac{1}{C_{l ; 2}^{2}} \sum_{l_{1} l_{2} l_{3}} C_{l_{1}}^{2} C_{l_{2}} C_{l_{3}}\left(\begin{array}{ccc}
l_{1} & l_{2} & l \\
0 & 0 & 0
\end{array}\right)^{2}\left(\begin{array}{ccc}
l_{1} & l_{3} & l \\
0 & 0 & 0
\end{array}\right)^{2} \frac{\left(2 l_{1}+1\right)\left(2 l_{2}+1\right)\left(2 l_{3}+1\right)}{(4 \pi)^{2}} \\
& \quad=\frac{1}{C_{l ; 2}^{2}} \sum_{l_{1} l_{2}}\left(2 l_{1}+1\right)\left(2 l_{2}+1\right) C_{l_{1}} C_{l_{2}}\left(\begin{array}{ccc}
l_{1} & l_{2} & l \\
0 & 0 & 0
\end{array}\right)^{2} \sum_{l_{3}} C_{l_{1}} C_{l_{3}}\left(\begin{array}{lll}
l_{1} & l_{3} & l \\
0 & 0 & 0
\end{array}\right)^{2} \frac{\left(2 l_{3}+1\right)}{(4 \pi)^{2}},
\end{aligned}
$$

which is larger than

$$
\begin{aligned}
& \frac{1}{C_{l ; 2}^{2}} \sum_{l_{2}}\left(2 l_{2}+1\right) C_{2} C_{l_{2}}\left(\begin{array}{ccc}
2 & l_{2} & l \\
0 & 0 & 0
\end{array}\right)^{2} \sum_{l_{3}} C_{2} C_{l_{3}}\left(\begin{array}{ccc}
2 & l_{3} & l \\
0 & 0 & 0
\end{array}\right)^{2} \frac{\left(2 l_{3}+1\right)}{(4 \pi)^{2}} \geq \frac{C_{2}^{2} C_{l+2}^{2}}{C_{l ; 2}^{2}} \sum_{l_{2}}\left(2 l_{2}+1\right) \\
& \quad \times\left(\begin{array}{ccc}
2 & l_{2} & l \\
0 & 0 & 0
\end{array}\right)^{2} \sum_{l_{3}}\left(\begin{array}{lll}
2 & l_{3} & l \\
0 & 0 & 0
\end{array}\right)^{2} \frac{\left(2 l_{3}+1\right)}{(4 \pi)^{2}}=\frac{C_{2}^{2} C_{l+2}^{2}}{C_{l ; 2}^{2}} .
\end{aligned}
$$

Now we have proved earlier that in the polynomial case, $C_{l ; 2} \simeq C_{l} \simeq l^{-\alpha}$, so the previous ratio does not converge to zero and $\hat{C}_{l ; 2}$ cannot be ergodic; the lower bound provided in the statement of the lemma follows from previous computations and easy manipulations.

For the second part of the statement, it is sufficient to note that

$$
\begin{aligned}
& \frac{1}{C_{l ; 2}^{2}} \sum_{l_{1} l_{2}}\left(2 l_{1}+1\right)\left(2 l_{2}+1\right) C_{l_{1}} C_{l_{2}}\left(\begin{array}{ccc}
l_{1} & l_{2} & l \\
0 & 0 & 0
\end{array}\right)^{2} \sum_{l_{3}} C_{l_{1}} C_{l_{3}}\left(\begin{array}{ccc}
l_{1} & l_{3} & l \\
0 & 0 & 0
\end{array}\right)^{2} \frac{\left(2 l_{3}+1\right)}{(4 \pi)^{2}} \\
& \leq \frac{\sup _{l_{1}}\left(2 l_{1}+1\right)^{-1} \sum_{l_{3}} \Gamma_{l_{1}} \Gamma_{l_{3}}\left\{C_{l_{1} 0 l_{3} 0}^{l 0}\right\}^{2}}{\sum_{l_{1} l_{3}} \Gamma_{l_{1}} \Gamma_{l_{3}}\left\{C_{l_{1} 0 l_{3} 0}^{l 0}\right\}^{2}} \leq \frac{\sup _{l_{1}} \sum_{l_{3}} \Gamma_{l_{1}} \Gamma_{l_{3}}\left\{C_{l_{1} 0 l_{3} 0}^{l 0}\right\}^{2}}{\sum_{l_{1} l_{3}} \Gamma_{l_{1}} \Gamma_{l_{3}}\left\{C_{l_{1} 0 l_{3} 0}^{l 0}\right\}^{2}}
\end{aligned}
$$

so the condition is met, just as for the standard case in Ref. 25.

Remarks:

(1) By inspection of the previous proof, we note that we have shown how the sufficient condition for asymptotic Gaussianity (HFG) is also such for ergodicity (HFE). More precisely, we have proved that

$$
\lim _{l \rightarrow \infty} \sup _{l_{1}} \frac{\sup _{l_{1}} \sum_{l} \Gamma_{l_{1}} \Gamma_{l_{2}}\left\{C_{l_{1} 0 l_{2} 0}^{l 0}\right\}^{2}}{\sum_{l_{1} l_{2}} \Gamma_{l_{1}} \Gamma_{l_{2}}\left\{C_{l_{1} 0 l_{2} 0}^{l 0}\right\}^{2}}=\lim _{l \rightarrow \infty} \sup _{\lambda} P\left(Z_{1}=l_{1} \mid Z_{2}=l_{2}\right)=0,
$$

where $\left\{Z_{l}\right\}$ is the Markov chain defined in Ref. 25 [Eqs. (57) and (58)], is a sufficient condition for the HFG (see Ref. 25, Proposition 9), and is also a sufficient condition to have $\lim _{l \rightarrow \infty} E\left\{\widetilde{C}_{l}-1\right\}^{2}=0$.

(2) In principle, the case $q=3$ can be dealt along similar lines.

(3) (On cosmic variance) Loosely speaking, the epistemological status of cosmological research has always been the object of some debate, as in some sense we are dealing with a science based on a single observation (our observed Universe). In the CMB community, this issue has been somewhat rephrased in terms of the so-called cosmic variance-i.e., it is taken as common knowledge that parameters relating only to lower multipoles (such as the value of $C_{l}$, for small values of $l$ ) are inevitably affected by an intrinsic uncertainty which cannot be eliminated (the variability due to the peculiar realization of the random field that we are able to observe), whereas this effect is taken to disappear at higher $l$ (implicitly assuming that something like the HFE should always hold). Our result seems to point out, apparently for the first time, the very profound role that the assumption of Gaussianity may play in this environment. In particular, for general non-Gaussian fields there is no guarantee that angular power spectra and related parameters can be consistently estimated even at high multipoles-i.e., the cosmic variance does not decrease at high frequencies for general nonGaussian models. 


\section{DISCUSSION AND DIRECTIONS FOR FURTHER RESEARCH}

This paper leaves many directions open for further research. We believe that the results of Secs. III and IV point out a very strong connection between conditions for HFE and HFG for isotropic spherical random fields. It is natural to suggest that equivalence may hold for Gaussian subordinated fields of any order $q$, or even more broadly for general Gaussian subordinated fields on homogeneous spaces of compact groups. Indeed, in this broader framework it is shown in Ref. 3 that independence of Fourier coefficients implies Gaussianity, which is the heuristic rationale behind our results here.

The connection between the HFE properties can also be studied under a different environment than Gaussian subordination. Consider, for instance, the class of completely random spherical fields, which was recently introduced in Refs. 8 and 9. Following the definition therein, we shall say that a spherical random field is completely random if for each $l$ we have that the vector $a_{l}$. $=\left(a_{l,-l}, \ldots, a_{l l}\right)$ is invariant with respect to the action of all matrices belonging to $\mathrm{SU}(2 l+1)$ and verifies $a_{l m}=(-1)^{m} \bar{a}_{l m}$. Because of this, the vector $a_{l}$ is clearly uniformly distributed on the manifold of random radius $\left\{\Sigma\left|a_{l m}\right|^{2}\right\}^{1 / 2}$ or, equivalently, introducing the $(2 l+1)$ vector $U_{l}$,

$$
U_{l}=\frac{1}{\sqrt{2 l+1}}\left\{\frac{\sqrt{2} \operatorname{Re} a_{l 1}}{\sqrt{\hat{C}_{l}}}, \frac{\sqrt{2} \operatorname{Re} a_{l 2}}{\sqrt{\hat{C}_{l}}}, \ldots, \frac{a_{l 0}}{\sqrt{\hat{C}_{l}}}, \frac{\sqrt{2} \operatorname{Im} a_{l 1}}{\sqrt{\hat{C}_{l}}}, \ldots, \frac{\sqrt{2} \operatorname{Im} a_{l l}}{\sqrt{\hat{C}_{l}}}\right\},
$$

law

it holds that, for $l$ large, it holds approximately that $U_{l} \sim U\left(S^{2 l}\right)$, i.e., $U_{l}$ it is asymptotically distributed on the unit sphere of $\mathbb{R}^{2 l+1}$. Under these conditions, it is simple to show that $\mathrm{HFE} \Rightarrow \mathrm{HFG}$, i.e.,

$$
\left\{\lim _{l \rightarrow \infty} E\left\{\tilde{C}_{l}-1\right\}^{2}=0\right\} \Rightarrow\left\{\frac{T_{l}(x)}{\sqrt{\operatorname{Var}\left(T_{l}\right)}} \stackrel{\text { law }}{\rightarrow} N(0,1) \quad \text { as } l \rightarrow \infty\right\} .
$$

Indeed, it is sufficient to note that, as before

$$
\frac{T_{l}(x)}{\sqrt{\operatorname{Var}\left(T_{l}\right)}}=\frac{T_{l}}{\sqrt{(2 l+1) C_{l}}}=\frac{\text { law }}{\sqrt{4 \pi} a_{l 0}}
$$

which we can write as

$$
\frac{a_{l 0}}{\sqrt{C_{l}}}=\frac{a_{l 0}}{\sqrt{\hat{C}_{l}}} \sqrt{\frac{\hat{C}_{l}}{C_{l}}}=\frac{a_{l 0}}{\sqrt{\hat{C}_{l}}} \sqrt{\widetilde{C}_{l}}
$$

Now, as $l \rightarrow \infty$

$$
\frac{a_{l 0}}{\sqrt{\hat{C}_{l}}} \rightarrow N(0,1)
$$

because the left hand side can be viewed as the marginal distribution for a uniform law on a sphere of growing dimension; the latter is asymptotically Gaussian, as a consequence of Poincaré lemma (see Ref. 11). We do not investigate this issue more fully here, and we leave for future research the determination of general conditions such that [cf. (32)]

$$
\text { the law of } U_{l} \text { and } U\left(S^{2 l}\right) \text { are asymptotically close as } l \rightarrow \infty .
$$

Obviously, for all fields such that (33) holds (i.e., those that are asymptotically completely random, to mimic the terminology in Refs. 8 and 9), by the same argument as before we have that 


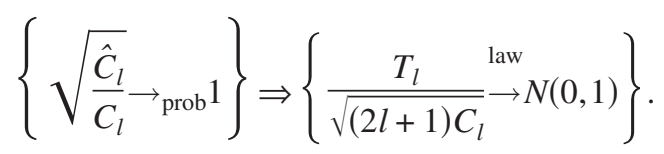

To conclude this work, we wish to provide two (somewhat pathological) examples where the HFE and HFG properties are indeed not equivalent. Consider first the (anisotropic) field

$$
h(x)=\sum_{l m} \xi_{l m} Y_{l m}(x), \quad \text { where } \xi_{l m}= \begin{cases}\xi_{l} & \text { for } m=0 \\ 0 & \text { otherwise }\end{cases}
$$

and the random variables $\xi_{l}$ verifies the assumption

$$
E \xi_{l}=0, \quad \sum_{l} E \xi_{l}^{2}<\infty \quad \text { and } E \xi_{l}^{4}<\infty .
$$

The field can be made isotropic by taking a random rotation $T(x)=h(g x)$, where $g$ is a random, uniformly distributed element of $\mathrm{SO}(3)$. We have as usual $T(x)=\Sigma_{l} \Sigma_{m=-l}^{l} a_{l m} Y_{l m}(x)$, where

$$
a_{l m}=\sum_{m^{\prime}=-l}^{l \text { law }} D_{m^{\prime} m}^{l}(g) \xi_{l m^{\prime}}=\sqrt{\frac{4 \pi}{2 l+1}} Y_{l m}(g) \xi_{l},
$$

and where $\left\{D^{l}(g)\right\}$ denotes the well-known Wigner representation matrices for $\mathrm{SO}(3)$, and the first identity in the law is discussed, for instance, in Refs. 2 and 27. Note that

$$
\sum_{m=-l}^{l}\left|a_{l m}\right|^{2}=\frac{4 \pi}{2 l+1} \xi_{l}^{2} \sum_{m=-l}^{l}\left|Y_{l m}(g)\right|^{2}=\xi_{l}^{2},
$$

as expected, because the sample angular power spectrum is invariant to rotations. Of course, in this case we do not have ergodicity, in general, i.e., it may happen that

$$
\frac{\sum_{m=-l}^{l}\left|a_{l m}\right|^{2}}{E \Sigma_{m=-l}^{l}\left|a_{l m}\right|^{2}}=\frac{\xi_{l}^{2}}{E \xi_{l}^{2}} \rightarrow 1
$$

and indeed for general sequences $\left\{\xi_{l}\right\}$

$$
E\left\{\frac{\xi_{l}^{2}}{E \xi_{l}^{2}}-1\right\}^{2}=E\left\{\frac{\xi_{l}^{2}}{E \xi_{l}^{2}}\right\}^{2}-1 \neq 0 .
$$

However, in the special case where

$$
\xi_{l}=\left\{\begin{array}{l}
e^{-l} \text { with probability } \frac{1}{2} \\
-e^{-l} \text { with probability } \frac{1}{2},
\end{array}\right.
$$

we obtain easily that $E\left\{\xi_{l}^{2} / E \xi_{l}^{2}-1\right\}^{2} \equiv 0$, while asymptotic Gaussianity fails. Hence, we have constructed an example where the HFE property holds but the HFG property does not. Note that the support of the vector $\left\{a_{l}\right\}$ is concentrated on a small subset of the sphere $S^{2 l}$; heuristically, this is what prevents Poincaré-type arguments to go through.

Now let $T(x)=\Sigma_{l} T_{l}(x)$ a mean-square continuous, isotropic Gaussian field, and define

$$
h(x):=\sum_{l} h_{l}(x)=\sum_{l} \eta_{l} T_{l}(x),
$$

where $\left\{\eta_{l}\right\}$ is a sequence of independent random variables such that $\left\{\eta_{l}\right\} \perp\left\{T_{l}(x)\right\}$ and 


$$
\eta_{l}=\left\{\begin{array}{l}
1 \text { w.p. } 1-\frac{1}{l^{2}} \\
l \text { w.p. } \frac{1}{2 l^{2}}, \text { whence } E \eta_{l}=1-1 / l^{2}, \quad E \eta_{l}^{2}=1, \text { and } E \eta_{l}^{4}=l^{2} . \\
-l \text { w.p. } \frac{1}{2 l^{2}}
\end{array}\right.
$$

It is trivial to verify that $\eta_{l} \rightarrow 1$ almost surely (apply the Borel-Cantelli lemma), the field $\{h(\cdot)\}$ is isotropic and mean-square continuous, and the HFG property holds, i.e., for any $x \in S^{2}$, as $l \rightarrow \infty$,

$$
\frac{h_{l}(x)}{\sqrt{\operatorname{Var}\left(h_{l}\right)}} \stackrel{\text { law }}{\rightarrow} N(0,1) \text {. }
$$

On the other hand,

$$
\operatorname{Var}\left\{\frac{\hat{C}_{l}}{C_{l}}\right\}=E \eta_{l}^{4} \times E\left\{\frac{\hat{C}_{l}}{C_{l}}\right\}^{2}-1=l^{2}\left\{1+\frac{2}{2 l+1}\right\}-1,
$$

whence the HFE clearly fails. Note, though, that here

$$
\frac{\hat{C}_{l}}{C_{l}} \rightarrow_{\text {a.s. }} 1 \text { as } l \rightarrow \infty
$$

that is, convergence in the almost sure sense holds, while convergence in the mean-square sense (which we used to define the HFE property) fails.

\section{ACKNOWLEDGMENTS}

We are grateful to Mirko D'Ovidio for some useful comments on an earlier version. We are also grateful to an anonymous referee for a careful reading of the manuscript, which led to great improvements in the presentation.

${ }^{1}$ Adler, R. J. and Taylor, J. E. T., Random Fields and Geometry (Springer, Berlin, 2007).

${ }^{2}$ Baldi, P. and Marinucci, D., "Some characterizations of the spherical harmonics coefficients for isotropic random fields," Stat. Probab. Lett. 77, 490 (2007).

${ }^{3}$ Baldi, P., Marinucci, D., and Varadarajan, V. S., "On the characterization of isotropic random fields on homogeneous spaces of compact groups," Electron. Commun. Probab. 12, 291 (2007).

${ }^{4}$ Bartolo, N., Komatsu, E., Matarrese, S., and Riotto, A., "Non-Gaussianity from inflation: Theory and observations," Phys. Rep. 402, 103 (2004).

${ }^{5}$ Biedenharn, L. C. and Louck, J. D., The Racah-Wigner Algebra in Quantum Theory, Encyclopedia of Mathematics and Its Applications Vol. 9 (Addison-Wesley, Reading, MA, 1981).

${ }^{6}$ Brockwell, P. J. and Davis, R. A., Time Series: Theory and Methods, Springer Series in Statistics, 2nd ed. (SpringerVerlag, New York, 1991).

${ }^{7}$ Cabella, P. and Marinucci, D., "Statistical challenges in the analysis of cosmic microwave background radiation," Ann. Appl. Stat. 3, 61 (2009).

${ }^{8}$ Dennis, M., "Canonical representation of spherical functions: Sylvester's theorem, Maxwell's multipoles and Majorana's sphere," J. Phys. A 37, 9487 (2004).

${ }^{9}$ Dennis, M., "Correlations between Maxwell's multipoles for Gaussian random functions on the sphere," J. Phys. A 38, 1653 (2005).

${ }^{10}$ Diaconis, P., Group Representations in Probability and Statistics, IMS Lecture Notes-Monograph Series No. 11 (IMS, Hayward, CA, 1988).

${ }^{11}$ Diaconis, P. and Freedman, D., "A dozen de Finetti-style results in search of a theory,” Ann. I.H.P. Probab. Stat. 23, 397 (1987).

${ }^{12}$ Dodelson, S., Modern Cosmology (Academic, New York, 2003).

${ }^{13}$ Efstathiou, G., "Myths and truths concerning estimation of power spectra: The case for a hybrid estimator," Mon. Not. R. Astron. Soc. 349, 603 (2004).

${ }^{14}$ Feller, W., An Introduction to Probability Theory and Its Applications, 2nd ed. (Wiley, New York, 1970), Vol. II.

${ }^{15}$ Guivarc'h, Y., Keane, M. and Roynette, B. Marches Aléatoires sul les Groupes de Lie, Lecture Notes in Mathematics Vol. 624 (Springer-Verlag, Berlin, 1977). 
${ }^{16} \mathrm{Hu}, \mathrm{W} .$, "The angular trispectrum of the CMB," Phys. Rev. D 64, 083005 (2001).

${ }^{17} \mathrm{Hu}$, Y. and Nualart, D., "Renormalized self-intersection local time for fractional Brownian motion," Ann. Probab. 33, 948 (2005).

${ }^{18}$ Janson, S., Gaussian Hilbert Spaces (Cambridge University Press, Cambridge, 1997).

${ }^{19}$ Kolb, E. and Turner, M., The Early Universe (Cambridge University Press, Cambridge, 1994).

${ }^{20}$ Komatsu, E., Dunkley, J., Nolta, M. R., Bennett, C. L., Gold, B., Hinshaw, G., Jarosik, N., Larson, D., Limon, M., Page, L., Spergel, D. N., Halpern, M., Hill, R. S., Kogut, A., Meyer, S. S., Tucker, G. S., Weiland, J. L., Wollack, E., and Wright, E. L., "Five-year Wilkinson microwave anisotropy probe observations: Cosmological Interpretation," Astrophys. J., Suppl. Ser. 180, 330 (2009).

${ }^{21}$ Leonenko, N., Limit Theorems for Random Fields with Singular Spectrum (Kluwer, Dordrecht, 1999).

${ }^{22}$ Liboff, R. L., Introductory Quantum Mechanics (Addison-Wesley, Reading, MA,1999).

${ }^{23}$ Marinucci, D., "A central limit theorem and higher order results for the angular bispectrum," Probab. Theory Relat. Fields 141, 389 (2008).

${ }^{24}$ Marinucci, D., "High-resolution asymptotics for the angular bispectrum of spherical random fields," Ann. Stat. 34, 1 (2006).

${ }^{25}$ Marinucci, D. and Peccati, G., "Group representations and high-resolution central limit theorems for subordinated spherical random fields," Bernoulli (in press).

${ }^{26}$ Marinucci, D. and Peccati, G., "High-frequency asymptotics for subordinated stationary fields on an Abelian compact group," Stochastic Proc. Appl. 118, 585 (2008).

${ }^{27}$ Marinucci, D. and Peccati, G., "Representations of SO(3) and angular polyspectra," J. Multivariate Anal. 101, 77 (2010).

${ }^{28}$ Marinucci, D. and Piccioni, M., "The empirical process on Gaussian spherical harmonics," Ann. Stat. 32, 1261 (2004).

${ }^{29}$ Nualart, D., The Malliavin Calculus and Related Topics, 2nd ed. (Springer, Berlin, 2006).

${ }^{30}$ Nualart, D. and Peccati, G., "Central limit theorems for sequences of multiple stochastic integrals," Ann. Probab. 33, 177 (2005).

${ }^{31}$ Nourdin, I. and Peccati, G., "Stein method on Wiener chaos," Probab. Theory Relat. Fields 145, 75 (2009).

${ }^{32}$ Nourdin, I., Peccati, G., and Reinert, G., "Invariance principles for homogeneous sums: Universality of the Gaussian Wiener chaos," Ann. Probab. (to appear).

${ }^{33}$ Peccati, G. and Pycke, J.-R., "Decompositions of stochastic processes based on irreducible group representations," Theory Probab. Applic. (to appear).

${ }^{34}$ Peccati, G. and Tudor, C. A., "Gaussian limits for vector-valued multiple stochastic integrals," Séminaire de Probabilités (Springer, Berlin, 2005), Vol. 38, pp. 247-262.

${ }^{35}$ Polenta, G., Marinucci, D., Balbi, A., De Bernardis, P., Hivon, E., Masi, S., Natoli, P., and Vittorio, N., "Unbiased estimation of angular power spectra," J. Cosmol. Astropart. Phys. 11, 1 (2005).

${ }^{36}$ Pycke, J.-R., “A decomposition for invariant tests of uniformity on the sphere," Proc. Am. Math. Soc. 135, 2983 (2007).

${ }^{37}$ Spergel, D. N., Bean, R., Doré, O., Nolta, M. R., Bennett, C. L., Dunkley, J., Hinshaw, G., Jarosik, N., Komatsu, E., Page, L., Peiris, H. V., Verde, L., Halpern, M., Hill, R. S., Kogut, A., Limon, M., Meyer, S. S., Odegard, N., Tucker, G. S., Weiland, J. L., Wollack, E., and Wright, E. L., "Three-year Wilkinson microwave anisotropy probe (WMAP) observations: Implications for cosmology,” Astrophys. J., Suppl. Ser. 170, 377 (2007).

${ }^{38}$ Spergel, D. N., Verde, L., Peiris, H. V., Komatsu, E., Nolta, M. R., Bennett, C. L., Halpern, M., Hinshaw, G., Jarosik, N., Kogut, A., Limon, M., Meyer, S. S., Page, L., Tucker, G. S., Weiland, J. L., Wollack, E., and Wright, E. L., "First-year Wilkinson microwave anisotropy probe (WMAP) observations: Determination of cosmological parameter," Astrophys. J., Suppl. Ser. 148, 175 (2003).

${ }^{39}$ Stein, E. M. and Weiss, G., Introduction to Fourier Analysis on Euclidean Spaces, Princeton Mathematical Series No. 32 (Princeton University Press, Princeton, NJ, 1971).

${ }^{40}$ Surgailis, D., "CLTs for polynomials of linear sequences: Diagram formula with illustrations," Theory and Applications of Long Range Dependence (Birkhäuser, Boston, Berlin, 2003), pp. 111-128.

${ }^{41}$ Varadarajan, V. S., An Introduction to Harmonic Analysis on Semisimple Lie Groups (Cambridge University Press, Cambridge, 1999), corrected reprint of the 1989 original.

${ }^{42}$ Varshalovich, D. A., Moskalev, A. N., and Khersonskii, V. K., Quantum Theory of Angular Momentum (World Scientific, Singapore, 1988).

${ }^{43}$ Vilenkin, N. J. and Klimyk, A. U., Representation of Lie Groups and Special Functions (Kluwer, Dordrecht, 1991).

${ }^{44}$ Wigman, I., "On the distribution of the nodal sets of random spherical harmonics," J. Math. Phys. 50, 013521 (2009a).

${ }^{45}$ Wigman, I., "Fluctuations of the nodal length of random spherical harmonics, preprint," e-print arXiv:0907.1648 (2009b).

${ }^{46}$ Yadav, A. P. S. and Wandelt, B. D., "Evidence of primordial non-Gaussianity (fNL) in the Wilkinson microwave anisotropy probe 3-year data at 2.8\%," Phys. Rev. Lett. 100, 181301 (2008).

${ }^{47}$ Yadrenko, M. I., Spectral Theory of Random Fields (Optimization Software, Inc., Boston, 1983), translated from the Russian (translation Series in Mathematics and Engineering). 